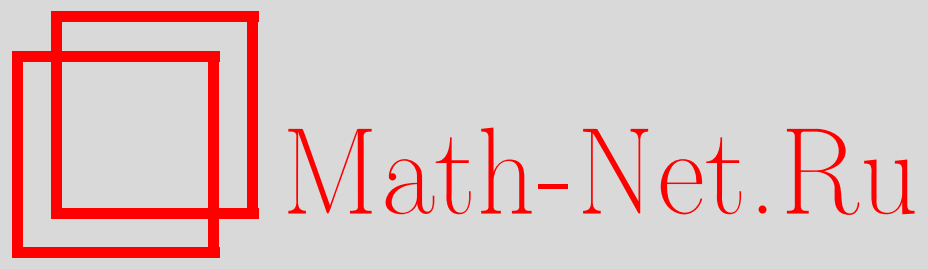

Д. Р. Яфаев, Оператор Шрёдингера: определители возмущения, функция спектрального сдвига, тождества следов и прочее, Функи. анализ и его прил., 2007, том 41, выпуск 3, 60-83

DOI: https://doi.org/10.4213/faa2868

Использование Общероссийского математического портала MathNet.Ru подразумевает, что вы прочитали и согласны с пользовательским соглашением

http://www . mathnet.ru/rus/agreement

Параметры загрузки:

IP : 107.22 .136 .117

26 апреля 2023 г., 02:44:25

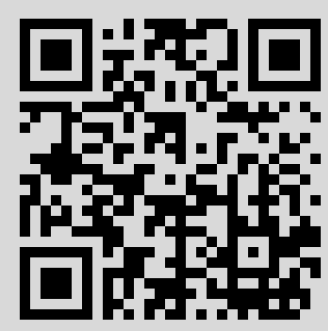




\title{
Оператор Шрёдингера: определители возмущения, функция спектрального сдвига, тождества следов и прочее
}

\author{
(C) 2007. Д. Р. ЯФАЕВ
}

Посвящается 100-летию со дня рождения Марка Григорьевича Крейна

\section{§1. Введение}

Для пары самосопряженных операторов $H_{0}, H$ в гильбертовом пространстве $\mathscr{H}$ функция спектрального сдвига $(\Phi С C) \xi(\lambda)=\xi\left(\lambda ; H, H_{0}\right)$ вводится соотношением

$$
\operatorname{Tr}\left(f(H)-f\left(H_{0}\right)\right)=\int_{-\infty}^{\infty} \xi(\lambda) f^{\prime}(\lambda) d \lambda,
$$

получившим название «формула следа». Концепция ФСС в теории возмущений появилась в литературе в работе [23] известного физика И. М. Лифшица. Ее математическая теория была вскоре построена М. Г. Крейном, установившим в [19] соотношение (1.1) для пары самосопряженных операторов $H_{0}, H$ с ядерной разностью $V=H-H_{0}$ и широкого класса функций $f$. Затем оно было обобщено им в [20] (более подробное изложение см. в [21]) на операторы $H_{0}, H$ с ядерной разностью $R(z)-R_{0}(z)$ их резольвент. Кроме того, в [3] была найдена связь (формула Бирмана-Крейна) с матрицей рассеяния (MP) $S(\lambda)=S\left(\lambda ; H, H_{0}\right)$. Там было показано, что для $\lambda$ из сердцевины (см. [33]) спектра оператора $H_{0}$

$$
\operatorname{Det} S(\lambda)=\exp (-2 \pi i \xi(\lambda)) \text {. }
$$

Позднее теория Крейна была развита в различных направлениях и применена к дифференциальным операторам. Достаточно подробное изложение «абстрактных» результатов можно найти в обзоре [5] или книге [33], где, однако, приложения к дифференциальным операторам почти не обсуждались. Настоящую работу можно до некоторой степени рассматривать как продолжение статьи [5] (а отчасти, и [6]). Однако здесь, в отличие от [5], изложение не является обзором. Мы рассматриваем только оператор Шрёдингера, являющийся достаточно представительным примером. Развиваемый ниже подход и основные полученные результаты являются новыми.

В $\S 2$ мы кратко напоминаем основные результаты теории Крейна. В $\S 3$ обсуждаются ее приложения к оператору Шрёдингера в пространстве $\mathscr{H}=$ $L_{2}\left(\mathbb{R}^{d}\right)$. Здесь наша основная цель состоит в распространении теории Крейна на возмущения неядерного типа. Интересные результаты в этом направлении можно найти в [18], [24] и [7]. Аналог ФСС называется регуляризованной ФСС. Наш «прямой» подход оказывается полезным и для возмущений ядерного типа и позволяет выявить специфические свойства ФСС, не выполняющиеся для случая произвольных самосопряженных операторов. Например, мы показываем, что $\xi(\lambda)$ - непрерывная функция переменной $\lambda$ при $\lambda>0$ (вообще говоря, 
она принадлежит только классу $L_{1}^{(l o c)}$ ), но доказательство этого факта оказывается довольно сложным при $d>3$.

Другая цель этой работы состоит в получении высокоэнергетического асимптотического разложения ФСС и связанных с ней объектов, что является существенным ингредиентом в выводе спектральных тождеств. Эти вопросы интенсивно изучались в литературе; см. [9], [12] по поводу одномерного случая и [8], [10] по поводу случая $d=3$. Мы в основном занимаемся многомерной задачей. В [8], [10] на потенциал $v(x)$ налагались довольно ограничительные предположения (требовалось чтобы он принадлежал классу Шварца). Отметим также работу [28] и обзор [29], где высокоэнергетическое асимптотическое разложение $Ф С С$ и спектральные тождества обсуждались в рамках микролокального анализа.

В настоящей работе мы предлагаем новый подход к построению асимптотического разложения $(\mathrm{AP})$ функции $\xi(\lambda)$ при $\lambda \rightarrow \infty$. При этом возникают две различные задачи. Первая из них - доказательство существования такого разложения. Вторая состоит в нахождении достаточно простых выражений для асимптотических коэффициентов. Первую задачу мы решаем в 6 , используя высокоэнергетическое асимптотическое разложение $\mathrm{MP} S(\lambda)$ (см. §5) и формулу Бирмана-Крейна. Это дает также $\mathrm{AP}$ функции $\operatorname{Tr}\left(R^{m}(z)-R_{0}^{m}(z)\right)$, где $m>d / 2-1$, при $|z| \rightarrow \infty$ во всей комплексной плоскости с разрезом по $\mathbb{R}_{+} \cdot \mathrm{B} \S 4$ мы используем другой метод построения этого АР, который работает только вдали от спектра, но зато дает эффективные выражения для асимптотических коэффициентов. Это приводит также к эффективным выражениям для коэффициентов в асимптотическом разложении ФСС. Другое выражение для этих коэффициентов получается в терминах инвариантов уравнения теплопроводности. Наконец, в $\S 7$ выводятся тождества следов как целого, так и полуцелого порядков.

\section{§2. Теория Крейна}

1. Ниже мы используем следующие стандартные определения (см., например, [11]): $\mathfrak{S}_{p}, p \geqslant 1,-$ симметрично нормированный идеал (с нормой $\left.\|\cdot\|_{p}\right)$ алгебры всех ограниченных операторов в гильбертовом пространстве $\mathscr{H}$; в частности, $\mathfrak{S}_{1}$ - идеал ядерных операторов, а $\mathfrak{S}_{2}$ - идеал операторов Гильберта-Шмидта; определитель $\operatorname{Det}(I+A)$ корректно определен для $A \in \mathfrak{S}_{1}$, и его свойства в существенном те же, что и в конечномерном случае. Для $A \in \mathfrak{S}_{p}, p=1,2, \ldots$, корректно определен регуляризованный определитель $\operatorname{Det}_{p}(I+A)$.

Пусть $H_{0}, H$ - самосопряженные операторы в гильбертовом пространстве $\mathscr{H}$, и пусть $R_{0}(z)=\left(H_{0}-z\right)^{-1}, R(z)=(H-z)^{-1}$ - их резольвенты, определенные соответственно на множествах $\varrho_{0}, \varrho$ регулярных точек операторов $H_{0}, H$. Определитель возмущения (OB) для пары $H_{0}, H$ с разностью $V=H-H_{0} \in \mathfrak{S}_{1}$ вводится соотношением

$$
D(z)=D_{H / H_{0}}(z)=\operatorname{Det}\left(I+V R_{0}(z)\right), \quad z \in \varrho_{0} .
$$

Функция $D(z)$ голоморфна на множестве $\varrho_{0}$,

$$
D(\bar{z})=\overline{D(z)}
$$

и она имеет корень $z$ порядка $k$ тогда и только тогда, когда $z$ является собственным значением кратности $k$ оператора $H$. Кроме того, $D(z)$ удовлетворяет 
тождеству

$$
D^{-1}(z) D^{\prime}(z)=\operatorname{Tr}\left(R_{0}(z)-R(z)\right), \quad z \in \varrho_{0} \cap \varrho .
$$

Эти свойства ОВ распространяются на случай $V R_{0}(z) \in \mathfrak{S}_{1}$.

$\Phi С С$ построена в терминах ОВ в следующей теореме М. Г. Крейна. Поскольку $D(z) \rightarrow 1$ при $|\operatorname{Im} z| \rightarrow \infty$, будем фиксировать ветвь функции $\ln D(z)$ в полуплоскостях $\pm \operatorname{Im} z>0$ условием $\arg D(z) \rightarrow 0$ при $|\operatorname{Im} z| \rightarrow \infty$.

Теорема 2.1. При $V \in \mathfrak{S}_{1}$ имеет место представление

$$
\ln D(z)=\int_{-\infty}^{\infty} \xi(\lambda)(\lambda-z)^{-1} d \lambda, \quad \operatorname{Im} z \neq 0,
$$

əəe

$$
\xi(\lambda)=\pi^{-1} \lim _{\varepsilon \rightarrow+0} \arg D(\lambda+i \varepsilon) .
$$

При почти всех (п.в.) $\lambda \in \mathbb{R}$ предел в (2.5) существует $и$

$$
\int_{-\infty}^{\infty}|\xi(\lambda)| d \lambda \leqslant\|V\|_{1}, \quad \int_{-\infty}^{\infty} \xi(\lambda) d \lambda=\operatorname{Tr} V .
$$

Кроме того, $\pm \xi(\lambda) \geqslant 0$, если $\pm V \geqslant 0$.

В лакунах непрерывного спектра $\xi(\lambda)$ связана со сдвигом собственных значений оператора $H$ относительно собственных значений оператора $H_{0}$.

Предложение 2.2. На составляющих интервалах множества $\varrho_{0} \cap \Phi С C$ $\xi(\lambda)$ принимает постоянные целочисленные значения. Если $\lambda$ - изолированное собственное значение кратности $k_{0}$ оператора $H_{0}$ u кратности $k$ оператора $H, \operatorname{mo} \xi(\lambda+0)-\xi(\lambda-0)=k_{0}-k$.

Формула следа (1.1) была обоснована М. Г. Крейном для достаточно широкого класса функций $f$.

Теорема 2.3. Пусть $V \in \mathfrak{S}_{1}$, а функиия $f$ непрерьвно дифферениируема $u$ ее производная допускает представление

$$
f^{\prime}(\lambda)=\int_{-\infty}^{\infty} \exp (-i t \lambda) d m(t), \quad|m|(\mathbb{R})<\infty,
$$

с конечной (комплексной) мерой $m$. Тогда

$$
f(H)-f\left(H_{0}\right) \in \mathfrak{S}_{1}
$$

и выполняется формула (1.1).

Замечание 2.4. Условие (2.6) может быть слегка модифицировано и ослаблено (см. [4], [25]). Например, в случае ограниченных операторов $H_{0}, H$ достаточно потребовать, чтобы $f^{\prime}$ была гельдеровски непрерывной функцией.

2. Напомним кратко основные положения теории рассеяния (детали см., например, в [33]). Обозначим через $E(\cdot)$ спектральный проектор оператора $H$, через $\mathscr{H}^{(a)}$ - его абсолютно непрерывное подпространство, а через $P$ - ортогональный проектор на $\mathscr{H}^{(a)}$; аналогичные объекты для оператора $H_{0}$ снабжаются индексом 0. Волновой оператор для пары самосопряженных операторов $H_{0}$ и $H$ определяется соотношением

$$
W_{ \pm}=W_{ \pm}\left(H, H_{0}\right)=\underset{t \rightarrow \pm \infty}{\mathrm{s}-\lim _{t \rightarrow \infty}} \exp (i H t) \exp \left(-i H_{0} t\right) P_{0}
$$


при условии, что этот сильный предел существует. Волновой оператор изометричен на $\mathscr{H}_{0}^{(a)}$ и обладает сплетающим свойством: $W_{ \pm} E_{0}(\Lambda)=E(\Lambda) W_{ \pm}$, где $\Lambda \subset \mathbb{R}$ - произвольное борелевское множество. В частности, его область значений $\operatorname{Ran} W_{ \pm}$лежит в $\mathscr{H}^{(a)}$. Оператор рассеяния

$$
\mathbf{S}=\mathbf{S}\left(H, H_{0}\right):=W_{+}^{*}\left(H, H_{0}\right) W_{-}\left(H, H_{0}\right)
$$

коммутирует с $H_{0}$. Оператор $W_{ \pm}$называется полным, если $\operatorname{Ran} W_{ \pm}=\mathscr{H}^{(a)}$. Таким образом, если волновые операторы $W_{ \pm}$существуют и полны, то абсолютно непрерывные части операторов $H_{0}$ и $H$ унитарно эквивалентны. В этом случае оператор рассеяния унитарен на пространстве $\mathscr{H}_{0}^{(a)}$.

При определении MP $S\left(\lambda ; H, H_{0}\right)$ предположим для простоты, что спектр $\sigma_{0}=\mathbb{C} \backslash \varrho_{0}$ оператора $H_{0}$ абсолютно непрерывен, состоит из конечного (или локально конечного) объединения замкнутых интервалов и имеет постоянную кратность. Пусть $\mathfrak{h}$ - вспомогательное гильбертово пространство, такое, что $\operatorname{dimh}$ равняется этой кратности. Тогда существует унитарное отображение $\mathscr{F}: \mathscr{H} \rightarrow L_{2}\left(\sigma_{0} ; \mathfrak{h}\right)$, для которого оператор $\mathscr{F} H_{0} \mathscr{F}^{*}$ действует как умножение на независимую переменную $(\lambda)$ в пространстве $L_{2}\left(\sigma_{0} ; \mathfrak{h}\right)$. Из соотношения $\mathbf{S} H_{0}=H_{0} \mathbf{S}$ следует, что оператор $\mathscr{F} \mathbf{S} \mathscr{F}^{*}$ действует в пространстве $L_{2}\left(\sigma_{0} ; \mathfrak{h}\right)$ как умножение на унитарную операторную функцию $S(\lambda)=S\left(\lambda ; H, H_{0}\right): \mathfrak{h} \rightarrow \mathfrak{h}$, определенную для п.в. $\lambda \in \sigma_{0}$. Конечно, это определение фиксирует MP $S(\lambda)$ с точностью до унитарной эквивалентности в пространстве $\mathfrak{h}$.

Теорема Като-Розенблюма (см., например, [33]) утверждает, что волновые операторы $W_{ \pm}\left(H, H_{0}\right)$ существуют и полны, если $V \in \mathfrak{S}_{1}$. Связь между ФСС и МР была найдена в [3].

Теорема 2.5. Если $V \in \mathfrak{S}_{1}$, то $S(\lambda)-I \in \mathfrak{S}_{1}$ и формула (1.2) выполняется для п.в. $\lambda \in \sigma_{0}$.

Соотношение (1.2) часто используется для определения (с точностью до целого числа) ФСС на абсолютно непрерывной части спектра. Формально дифференцируя (1.2), получим, что

$$
\operatorname{Tr}\left(S^{*}(\lambda) S^{\prime}(\lambda)\right)=-2 \pi i \xi^{\prime}(\lambda) .
$$

Это соотношение иногда удобнее в применениях, чем (1.2), поскольку существует простое выражение для следа интегрального оператора с гладким ядром.

3. Ввиду наших приложений к оператору Шрёдингера обсудим обобщения теорем 2.1, 2.3 и 2.5, приспособленные к полуограниченному случаю. За счет сдвига на константу можно считать, что операторы $H_{0}+c I$ и $H+c I$ положительно определены. Будем предполагать, что

$$
R^{m}(z)-R_{0}^{m}(z) \in \mathfrak{S}_{1}
$$

для $z=-c$ и некоторого $m>0$. Из теоремы 2.1 следует, что ФСС $\xi\left(\mu ; h, h_{0}\right)$ для пары $h_{0}=\left(H_{0}+c I\right)^{-m}, h=(H+c I)^{-m}$ существует и принадлежит пространству $L_{1}(\mathbb{R})$. Кроме того, согласно предложению 2.2 , она равняется нулю на $\mathbb{R}_{-}$и для достаточно больших $\mu>0$. Для исходной пары $H_{0}, H$ ФСС определяется соотношениями

$$
\xi\left(\lambda ; H, H_{0}\right)=-\xi\left((\lambda+c)^{-m} ;(H+c I)^{-m},\left(H_{0}+c I\right)^{-m}\right)
$$


для $\lambda>-c$ и $\xi\left(\lambda ; H, H_{0}\right)=0$ для $\lambda \leqslant-c$. В терминах $\mathrm{OB} D_{h / h_{0}}(\zeta)$ для пары $h_{0}, h$ ФСС строится по формуле

$$
\xi\left(\lambda ; H, H_{0}\right)=\pi^{-1} \arg D_{h / h_{0}}\left((\lambda+c+i 0)^{-m}\right) .
$$

Класс допустимых функций получается из теоремы 2.3 (см. также замечание 2.4) заменой переменных $\mu=(\lambda+c)^{-m}$. Из принципа инвариантности Бирмана (см., например, [33]) вытекает, что при условии (2.10), где $z=-c$, волновые операторы $W_{ \pm}\left(H, H_{0}\right)$ существуют и полны. В этом случае МР связаны соотношением $S\left(\lambda ; H, H_{0}\right)=S^{*}\left(\mu ; h, h_{0}\right)$. Таким образом, следующий результат является прямым следствием теорем 2.1, 2.3 и 2.5 .

Теорема 2.6. Пусть условие (2.10) выполняется для $z=-$. Определим $\Phi C C \xi(\lambda)=\xi\left(\lambda ; H, H_{0}\right)$ равенствами (2.11) для $\lambda>-c u \xi(\lambda)=0$ для $\lambda \leqslant-c$. Она удовлетворяет соотношению

$$
\int_{-\infty}^{\infty}|\xi(\lambda)|(1+|\lambda|)^{-m-1} d \lambda<\infty
$$

и связана с МР равенством (1.2). Предположим, что функиия $f$ имеет две локально ограниченные производные и

$$
\left(\lambda^{m+1} f^{\prime}(\lambda)\right)^{\prime}=O\left(\lambda^{-1-\varepsilon}\right), \quad \varepsilon>0, \lambda \rightarrow \infty .
$$

Тогда выполняются включение (2.7) и формула следа (1.1).

Следствие 2.7. Формула следа (1.1) справедлива для всех функщий $f(\lambda)=$ $(\lambda-z)^{-k}$, где $k \geqslant m u z \in \varrho_{0} \cap \varrho$. В частности,

$$
\operatorname{Tr}\left(R^{m}(z)-R_{0}^{m}(z)\right)=-m \int_{-\infty}^{\infty} \xi(\lambda)(\lambda-z)^{-m-1} d \lambda .
$$

Замечание 2.8. Если условие (2.10) верно для $m=1$, то обобщенный ОВ

$$
\widetilde{D}_{-c}(z):=\operatorname{Det}\left(I+(z+c) R(-c) V R_{0}(z)\right)=D_{h / h_{0}}\left((z+c)^{-1}\right)
$$

корректно определен и удовлетворяет уравнению (2.3). Зафиксируем непрерывную ветвь $\arg \widetilde{D}_{-c}(z)$ условием $\arg \widetilde{D}_{-c}(-c)=0$. Тогда из формулы $(2.12)$ следует, что

$$
\xi\left(\lambda ; H, H_{0}\right)=\pi^{-1} \arg \widetilde{D}_{-c}(\lambda+i 0) .
$$

ФСС имеет определенный знак для знакоопределенных возмущений оператора $H_{0}$. Мы понимаем знак возмущения в смысле квадратичных форм. Следующий результат получен в [17] (см. также теорему 8.10 .3 в [33]).

Теорема 2.9. Пусть выполняется условие (2.10). Тогда $\xi(\lambda) \geqslant 0$, если $H \geqslant$ $H_{0}, u \xi(\lambda) \leqslant 0$, если $H \leqslant H_{0}$.

4. В приложениях к дифференциальным операторам удобно использовать понятие регуляризованного определителя и ввести регуляризованный ОВ

$$
D_{p}(z)=\operatorname{Det}_{p}\left(I+V R_{0}(z)\right), \quad p=2,3, \ldots
$$

Это определение корректно при $V R_{0}(z) \in \mathfrak{S}_{p}$. В этом случае функция $D_{p}(z)$ голоморфна на множестве $\varrho_{0}$. Многие свойства обычных ОВ переносятся на 
регуляризованные ОВ. Так, тождество (2.2) остается верным, а обобщение (2.3) имеет вид

$$
D_{p}^{-1}(z) D_{p}^{\prime}(z)=-\operatorname{Tr}\left(R(z)-\sum_{k=0}^{p-1}(-1)^{k} R_{0}(z)\left(V R_{0}(z)\right)^{k}\right) .
$$

\section{§3. Регуляризованный ОВ и ФСС для оператора Шрёдингера}

1. Ниже мы рассматриваем пару самосопряженных операторов $H_{0}=-\Delta$,

$$
H=-\Delta+v(x), \quad v(x)=\overline{v(x)},
$$

в пространстве $\mathscr{H}=L_{2}\left(\mathbb{R}^{d}\right)$. Предполагается, что потенциал $v(x)$ достаточно быстро убывает на бесконечности, т. е.

$$
|v(x)| \leqslant C(1+|x|)^{-\rho},
$$

где по крайней мере $\rho>1$. Тогда положительный спектр оператора $H$ абсолютно непрерывен, а его отрицательный спектр состоит из собственных значений $\lambda_{1}<\lambda_{2} \leqslant \lambda_{3} \leqslant \ldots$, перенумерованных со своими кратностями (см, например, [27]); точка $\lambda=0$ также может быть собственным значением оператора $H$. Волновые операторы (2.8) существуют и полны, так что $\operatorname{Ran} W_{ \pm}=E\left(\mathbb{R}_{+}\right) \mathscr{H}$ (см., например, [22]).

Каноническая диагонализация оператора $H_{0}$ осуществляется оператором $\mathscr{F}$ : $\mathscr{H} \rightarrow L_{2}\left(\mathbb{R}_{+} ; L_{2}\left(\mathbb{S}^{d-1}\right)\right)$, определяемым соотношением $(\mathscr{F} u)(\lambda ; \omega)=\left(\Gamma_{0}(\lambda) u\right)(\omega)$, где

$$
\left(\Gamma_{0}(\lambda) u\right)(\omega)=2^{-1 / 2} \lambda^{(d-2) / 4} \hat{u}\left(\lambda^{1 / 2} \omega\right), \quad \lambda>0, \omega \in \mathbb{S}^{d-1},
$$

а $\hat{u}$ - преобразование Фурье функции $u$. MP $S(\lambda)=S\left(\lambda ; H, H_{0}\right)$ корректно определена и является унитарным оператором в пространстве $L_{2}\left(\mathbb{S}^{d-1}\right)$ для п.в. $\lambda \in \mathbb{R}_{+}$. Кроме того, она допускает представление

$$
S(\lambda)=I-2 \pi i\left(\mathscr{A}_{0}(\lambda)+\widetilde{\mathscr{A}}(\lambda)\right),
$$

где

$$
\mathscr{A}_{0}(\lambda)=\Gamma_{0}(\lambda) V \Gamma_{0}^{*}(\lambda), \quad \widetilde{\mathscr{A}}(\lambda)=-\Gamma_{0}(\lambda) V R(\lambda+i 0) V \Gamma_{0}^{*}(\lambda) .
$$

Эти операторы корректно определены. Действительно, положим $\langle x\rangle^{-r}=(1+$ $\left.|x|^{2}\right)^{-r / 2}$ (мы пользуемся общим обозначением для функции и оператора умножения на нее) и выберем некоторое $r>1 / 2$. Тогда, согласно теореме Соболева, оператор $\Gamma_{0}(\lambda)\langle x\rangle^{-r}: \mathscr{H} \rightarrow L_{2}\left(\mathbb{S}^{d-1}\right)$ компактен и непрерывно зависит от $\lambda>0$. Принцип предельного поглощения утверждает, что операторная функция $\langle x\rangle^{-r} R_{0}(z)\langle x\rangle^{-r}$ непрерывна в комплексной плоскости вплоть до разреза по $[0, \infty)$ за возможным исключением точки $z=0$. Таким образом, при $\rho>1$ операторы (3.4) могут быть представлены в виде произведения ограниченных операторов, непрерывно зависящих от $\lambda>0$. Отсюда следует, что MP $S(\lambda)-$ непрерывная (в операторной топологии) функция при $\lambda>0$.

Если оценка (3.2) выполнена при $\rho>d$, то включение (2.10) выполняется для всех $z \in \varrho$ и любого положительного целого числа $m>d / 2-1$. Этот результат - непосредствнное следствие резольвентного тождества и включения $\langle x\rangle^{-\rho / 2} R_{0}(z) \in \mathfrak{S}_{2}$, если $m=1$, но требует некоторых ухищрений, если $m>1$ (см. [32] или [27]). Таким образом, следующее утверждение вытекает из теорем 2.6 и 2.9 . 
Теорема 3.1. Предположим, что условие (3.2) выполняется при $\rho>d$. Пусть с $>-\lambda_{1}, m$ - положительное целое число и $2(m+1)>d$. Определим ФСС равенством (2.11) для $\lambda>-c$ и положим $\xi\left(\lambda ; H, H_{0}\right)=0$ для $\lambda \leqslant-c$. Тогда выполняется условие (2.13),

$$
\xi(\lambda)=0 \quad \partial л я \lambda<\lambda_{1}, \quad \xi(\lambda)=-n \quad \partial \Omega я \lambda \in\left(\lambda_{n}, \lambda_{n+1}\right), \quad \lambda_{n}<\lambda_{n+1},
$$

и ФСС связана с МР равенством (1.2) при $\lambda>0$. Если функиия $f$ имеет две локально ограниченные производные и удовлетворяет условию (2.14), то справедливы включение (2.7) и формула следа (1.1) (в частности, (2.15)). Кроме того, если $v(x) \geqslant 0(v(x) \leqslant 0)$, то $\xi(\lambda) \geqslant 0$ (соответственно $\xi(\lambda) \leqslant 0)$.

Следствие 3.2. Пусть $d \leqslant 3 u \rho>d$. Тогда включение (2.10) и формула (2.15) справедливы для $m=1$, а ФСС может быть выражена через обобщенный $О В$ (2.16) соотношением (2.17).

2. Теперь мы изложим прямой подход к построению ФСС для многомерного оператора Шрёдингера (3.1). Это позволяет изучить некоторые конкретные свойства функции $\xi(\lambda)$, такие, как непрерывность при $\lambda>0$ и поведение при $\lambda \rightarrow+\infty$ и $\lambda \rightarrow 0$. Мы предполагаем здесь, что условие (3.2) выполняется по крайней мере для $\rho>1$, но не обязательно для $\rho>d$, как в ядерном методе. На самом деле $\rho$ зависит от размерности $d$ задачи и различно в различных утверждениях. Для основных результатов мы предполагаем, что $\rho>2$. В этом случае оператор $H$ может иметь только конечное число $\mathrm{N}$ отрицательных собственных значений. Таким образом, прямой подход позволяет построить ФСС вне рамок ядерного метода.

В предыдущем параграфе ФСС определялась соотношением (2.12), которое не всегда удобно. В одномерном случае $V R_{0}(z) \in \mathfrak{S}_{1}$, так что $\Phi C C$ может быть выражена по формуле (2.5) через ОВ $D(z)=D_{1}(z)$ для исходной пары $H_{0}, H$. При $d>1$ аналогичное представление получается в терминах регуляризованного ОВ $D_{p}(z)$, определяемого формулой (2.18). Структура ОВ $D_{p}(z)$ усложняется с ростом $p$, и обычно из какого-либо свойства $D_{p}(z)$ вытекает аналогичное свойство ОВ для бо́льших $p$. Поэтому естественно выбирать наименьшее $p$, такое, что $V R_{0}(z) \in \mathfrak{S}_{p}$.

Следующее элементарное утверждение показывает, что свойства регуляризованных ОВ в существенном те же, что и в ядерном случае.

Предложение 3.3. Предположим, что $\rho>1$, если $d=1,2, \rho>3 / 2$, если $d=3, u \rho \geqslant 2$, если $d \geqslant 4$. Пусть $p=1$ при $d=1, p=2$ при $d=2,3 u$ $p>d / 2$ при $d \geqslant 4$. Тогда функиия $D_{p}(z)$ аналитична в комплексной плоскости с разрезом по положительной полуоси $[0, \infty)$ и удовлетворяет тождествам (2.2) и (2.19). Эта функиия может иметь нули только на отрицательной полуоси, где они совпадают с собственными значениями оператора $H$; порядок ее нуля равняется кратности соответствующего собственного значения.

Пусть $r=\rho / 2, G=\langle x\rangle^{-r}$, и пусть $\mathscr{V}$ - оператор умножения на ограниченную функцию $\langle x\rangle^{\rho} v(x)$. Поскольку ненулевые собственные числа операторов $V R_{0}(z)$ и $G R_{0}(z) G \mathscr{V}$ совпадают, то

$$
D_{p}(z)=\operatorname{Det}_{p}\left(I+G R_{0}(z) G \mathscr{V}\right) .
$$


Это представление более удобно, чем (2.18), поскольку операторная функция $G R_{0}(z) G$ непрерывна вплоть до разреза в классах $\mathfrak{S}_{p}$ для достаточно больших $p$. В следующем утверждении используется тот факт, что если $D_{p}(\lambda \pm i 0)=0$ при $\lambda>0$, то -1 является собственным числом компактного оператора $G R_{0}(\lambda \pm i 0) G \mathscr{V}$ и, следовательно, $\lambda$ - собственное значение оператора $H$. Однако в силу теоремы Като (см., например, [27]) этот оператор не имеет положительных собственных значений.

Предложение 3.4. Пусть $\rho>1$, если $d=1, \rho>3 / 2$, если $d=2, u \rho>2$, если $d \geqslant 3$. Положим $p=1$ для $d=1, p=2$ для $d=2,3$ u $p=d$ для $d \geqslant 4$. Тогда бункиия $D_{p}(z)$ непрерывна вплоть до разреза $[0, \infty)$ за возможным исключением точки $z=0$. Функиия $D_{p}(\lambda \pm i 0)$ не имеет нулей при $\lambda>0$.

Что касается высокоэнергетической асимптотики ОВ, то имеет место следующее

Предложение 3.5. В условиях предложения 3.4

$$
\lim _{|z| \rightarrow \infty} D_{p}(z)=1
$$

равномерно по $\arg z \in[0,2 \pi]$.

При доказательстве ограничимся случаем $d=3$. Воспользуемся тождеством

$$
\operatorname{Det}_{2}\left(I+G R_{0}(z) G \mathscr{V}\right)=\operatorname{Det}_{3}\left(I+G R_{0}(z) G \mathscr{V}\right) \exp \left(-2^{-1} \operatorname{Tr}\left(V R_{0}(z)\right)^{2}\right)
$$

Заметим, что

$$
\left\|G R_{0}(z) G\right\|_{3}^{3} \leqslant\left\|G R_{0}(z) G\right\|\left\|G R_{0}(z) G\right\|_{2}^{2} .
$$

Первый сомножитель в правой части стремится к нулю при $|z| \rightarrow \infty$, а из явной формулы для интегрального ядра оператора $R_{0}(z)$ следует, что второй сомножитель равномерно ограничен. Поэтому первый сомножитель в правой части равенства (3.7) стремится к 1 при $|z| \rightarrow \infty$. То же самое верно для второго сомножителя, потому что

$$
\operatorname{Tr}\left(V R_{0}(z)\right)^{2}=(4 \pi)^{-2} \int_{\mathbb{R}^{3}} \int_{\mathbb{R}^{3}} v(x) v(y)|x-y|^{-2} e^{2 i \sqrt{z}|x-y|} d x d y,
$$

и интеграл стремится к нулю в силу леммы Римана-Лебега.

ОВ $D_{p}(z)$ сингулярен в точке $z=0$ только при $d=1$ и $d=2$.

Предложение 3.6. Пусть $d \geqslant 3 u \rho>2$. Положим $p=2$ nри $d=3 u$ $p=d$ при $d \geqslant 4$. Тогда $O B D_{p}(0)$ корректно определен $u D_{p}(z) \rightarrow D_{p}(0)$, когда $|z| \rightarrow 0$.

Ясно, что $D_{p}(0)=0$ тогда и только тогда, когда -1 является собственным числом компактного оператора $G R_{0}(0) G \mathscr{V}$. В этом случае говорят, что оператор $H$ имеет резонанс при нулевой энергии (в частности, $H$ может иметь нулевое собственное значение).

Мы избегаем изучения ОВ $D_{p}(z)$ при $|z| \rightarrow 0$, налагая слабое априорное предположение

$$
\lim _{|z| \rightarrow 0}|z|^{\alpha} \ln D_{p}(z)=0
$$

для подходящего $\alpha>0$. Конечно, в случае $d \geqslant 3$ условие (3.8) выполняется для всех $\alpha>0$, если $D_{p}(0) \neq 0$. Впрочем, условие (3.8) может быть выведено из низкоэнергетического (при $z \rightarrow 0$ ) разложения резольвенты $R(z)$ (см. [15] для $d=3$ ), что требует, однако, более сильного условия, чем $\rho>2$. 
3. Мы считаем функцию $\arg D_{p}(z)$ непрерывной в комплексной плоскости с разрезом по $\left[\lambda_{1}, \infty\right)$, что возможно, поскольку там $D_{p}(z) \neq 0$. Согласно $(3.6)$, ветвь этой функции фиксируется условием $\arg D_{p}(z) \rightarrow 0$ при $|z| \rightarrow \infty$.

Построим теперь регуляризованную ФСС $\xi_{p}$.

Теорема 3.7. Пусть условие (3.2) выполняется при $\rho>2$, и пусть $p=1$ для $d=1, p=2$ для $d=2,3$ u $p=d$ для $d \geqslant 4$. Предположим, что (3.8) справедливо при $\alpha=1$. Определим регуляризованную ФСС $\xi_{p}$ равенством (2.5) в терминах регуляризованного $O B D_{p}(z)$. Тогда представление $(2.4)$ для $\ln D_{p}(z)$ выполняется с функиией $\xi_{p}(\lambda)$. Функиия $\xi_{p}$ удовлетворяет равенствам (3.5) при $\lambda<0$. Она непрерьвна при $\lambda>0, \xi_{p}(\lambda)=o(1)$ nри $\lambda \rightarrow \infty, \xi_{p}(\lambda)=o\left(\lambda^{-1}\right)$ при $\lambda \rightarrow 0$, а интеграль от $\xi_{p}(\lambda) \lambda^{-1}$ u $\xi_{p}(\lambda)$ сходятся (но не обязательно абсолютно) соответственно на бесконечности и в точке $\lambda=0$.

Действительно, функция $\ln D_{p}(z)$ аналитична в комплексной плоскости с разрезом по $\left[\lambda_{1}, \infty\right)$ и непрерывна вплоть до разреза за исключением точек $\lambda_{1}, \ldots, \lambda_{\mathrm{N}}$ и, возможно, нуля. Рассмотрим в комплексной плоскости замкнутый контур $\Gamma_{R, \varepsilon}$, состоящий из интервалов $\left(\lambda_{1}, R+i 0\right)$ и $\left(R-i 0, \lambda_{1}\right)$, лежащих на верхнем и нижнем берегах разреза, и окружности $C_{R}$ радиуса $R$, проходимой против часовой стрелки. Кроме того, мы обходим каждую точку $\lambda_{j}$, $j=1, \ldots, \mathrm{N}$, и точку 0 по полуокружностям $C_{\varepsilon}^{ \pm}\left(\lambda_{j}\right)$ и $C_{\varepsilon}^{ \pm}(0)$ радиуса $\varepsilon$. В силу теоремы Коши для произвольного комплексного $z$ при достаточно малом $\varepsilon$ и достаточно большом $R$

$$
\ln D_{p}(z)=(2 \pi i)^{-1} \int_{\Gamma_{R, \varepsilon}} \ln D_{p}(\zeta)(\zeta-z)^{-1} d \zeta
$$

Согласно (3.6), интеграл по $C_{R}$ стремится к нулю при $R \rightarrow \infty$. Поскольку функция $D(z)$ имеет только нули конечного порядка в точках $\lambda_{1}, \ldots, \lambda_{\mathrm{N}}$, интегралы по $C_{\varepsilon}^{ \pm}\left(\lambda_{j}\right)$ стремятся к нулю при $\varepsilon \rightarrow 0$. Интегралы по $C_{\varepsilon}^{ \pm}(0)$ стремятся к нулю при $\varepsilon \rightarrow 0$ в силу условия (3.8), где $\alpha=1$. Таким образом, представление $(2.4)$ для $D_{p}(z)$ с функцией $\xi_{p}(\lambda)$, определяемой формулой $(2.5)$, следует из уравнений $(2.2)$ и $(3.9)$.

Получим теперь формулу следа.

Теорема 3.8. Пусть $f$ - ограниченная рациональная функиия с невещественными полюсами. Тогда в условиях теоремы 3.7

$$
\operatorname{Tr}\left(f(H)-\left.\sum_{k=0}^{p-1} \frac{1}{k !} \frac{d^{k} f\left(H_{0}+\varepsilon V\right)}{d \varepsilon^{k}}\right|_{\varepsilon=0}\right)=\int_{-\infty}^{\infty} \xi_{p}(\lambda) f^{\prime}(\lambda) d \lambda
$$

u, в частности,

$$
\operatorname{Tr}\left(R(z)-\sum_{k=0}^{p-1}(-1)^{k} R_{0}(z)\left(V R_{0}(z)\right)^{k}\right)=-\int_{-\infty}^{\infty} \xi_{p}(\lambda)(\lambda-z)^{-2} d \lambda .
$$

Действительно, продифференцируем представление вида (2.4) для $D_{p}(z)$. Учитывая формулу (2.19), мы получим сначала (3.11), что дает (3.10) для $f(\lambda)=(\lambda-z)^{-1}, \operatorname{Im} z \neq 0$. Затем, дифференцируя (3.11), распространим формулу (3.10) на функции $f(\lambda)=(\lambda-z)^{-m}$, где $m=2,3, \ldots$. Ясно, что формула (3.10) остается верной для линейных комбинаций этих функций. 
Формула следа (1.1), конечно, не имеет смысла, если $\rho \leqslant d$, так что представление (3.10) может рассматриваться как ее регуляризация. Если $d=1$, то $\xi=\xi_{1}$ и теорема 3.8 содержится в теореме 2.6 .

Применяя принцип аргумента к функции $D_{p}(z)$, получим тождество следа нулевого порядка.

Теорема 3.9. В условиях предложения 3.6 предположим, что $D_{p}(0) \neq 0$. тогда

$$
\arg D_{p}(\infty)-\arg D_{p}(0)=\pi \mathrm{N},
$$

где $\mathrm{N}$ - общее число отрицательных собственных значений оператора $H$.

Подчеркнем, что, согласно определению (2.5), при условии $D_{p}(0) \neq 0$ предел $\xi_{p}(+0)$ существует. Формула (3.12) (называемая формулой Левинсона) означает, что регуляризованная $\Phi С С \xi_{p}(\lambda)$ непрерывна в точке $\lambda=0$. В случаях $d=1$ и $d=2$ нужно учитывать сингулярность ОВ $D_{p}(z)$ в точке $z=0$.

При положительных $\lambda$ регуляризованная ФСС связана с MP $S(\lambda)$. Относительно обозримое соотношение получается только при $p=2$. Пусть оператор $\mathscr{A}_{0}(\lambda)$ (называемый первым борновским приближением к МР) определяется формулой (3.4). Он самосопряжен, не зависит от резольвенты оператора $H$ и строится непосредственно в терминах преобразования Фурье потенциала $v$. Положим

$$
\nu(\lambda)=\operatorname{Tr}\left(S(\lambda)-I+2 \pi i \mathscr{A}_{0}(\lambda)\right)=-2 \pi i \operatorname{Tr} \widetilde{\mathscr{A}}(\lambda) .
$$

Следующий результат может рассматриваться как модификация формулы Бирмана-Крейна (1.2). Его доказательство основано на тождестве

$$
\begin{aligned}
& \operatorname{Det}_{2}\left(I-(V-V R(z) V)\left(R_{0}(z)-R_{0}(\bar{z})\right)\right) \\
& \quad=D_{2}(\bar{z}) D_{2}(z)^{-1} \exp \left(-\operatorname{Tr}\left(V R(z) V\left(R_{0}(z)-R_{0}(\bar{z})\right)\right)\right),
\end{aligned}
$$

где мы переходим к пределу при $z \rightarrow \lambda+i 0$.

Теорема 3.10. Пусть условие (3.2) выполнено при $\rho>3 / 2$, если $d=2, u$ при $\rho>2$, если $d=3$. Тогда бункиия $\xi_{2}(\lambda)$ связана с $M P S(\lambda)$ формулой

$$
\operatorname{Det}_{2} S(\lambda)=e^{-2 \pi i \xi_{2}(\lambda)-\nu(\lambda)}, \quad \lambda>0 \text {. }
$$

4. Предположим, что $v$ удовлетворяет условию (3.2) при $\rho>d$. Тогда включение $(2.10)$ выполняется при $2(m+1)>d$, и обычная ФСС $\xi(\lambda)$ определяется соотношением (2.11). Найдем связь между $\xi$ и регуляризованной ФСС $\xi_{p}$, что позволит получить новую информацию о ФСС $\xi$, дополняющую результаты разд. 1.

Пусть сначала $d \leqslant 3$, так что $m=1$. Соотношение между обобщенным ОВ $\widetilde{D}(z)=\widetilde{D}_{-c}(z),-c<\lambda_{1}$, определенным равенством $(2.16)$, и регуляризованным OB $D_{2}(z)$ легко получается сопоставлением уравнений $(2.3)$ для $\widetilde{D}(z)$ и $(2.19)$ для $D_{2}(z)$.

Предложение 3.11. Если $d=2$ или $d=3$ и условие (3.2) выполняется для $\rho>d$, mo

$$
\widetilde{D}(z)=D_{2}^{-1}(-c) D_{2}(z) \exp \left((4 \pi)^{-1} \int_{\mathbb{R}^{d}} v(x) d x b_{d}(z)\right),
$$

где $b_{2}(z)=-\ln (-z / c), b_{3}(z)=c^{1 / 2}-(-z)^{1 / 2} u \arg (-z) \in(-\pi, \pi)$. 
Сравнивая аргументы обеих частей формулы (3.13) и переходя к пределу при $z \rightarrow \lambda+i 0$, получим соотношение между $\Phi С С ~ \xi(\lambda)$, которая может быть определена формулой $(2.17)$, и $\xi_{2}(\lambda)$. Затем мы используем теоремы 3.7 и 3.9.

Теорема 3.12. Пусть выполняются условия предложения 3.11. Тогда при $\lambda>0 \Phi C C \xi(\lambda)$ допускает представление

$$
\xi(\lambda)=\xi_{2}(\lambda)+2^{-1}(2 \pi)^{-d}\left|\mathbb{S}^{d-1}\right| \int_{\mathbb{R}^{d}} v(x) d x \lambda^{(d-2) / 2} .
$$

Функиия $\xi(\lambda)$ непрерывна при $\lambda>0 u$

$$
\xi(\lambda)=2^{-1}(2 \pi)^{-d}\left|\mathbb{S}^{d-1}\right| \int_{\mathbb{R}^{d}} v(x) d x \lambda^{(d-2) / 2}+o(1), \quad \lambda \rightarrow \infty .
$$

Eсли $d=3$ и оператор $H$ не имеет резонанса при нулевой энергии, то существует предел $\xi(+0)=-\pi \mathrm{N}$, так что ФСС непрерывна в точке $\lambda=0$.

Если $d=1$, то $D_{2}(z)$ связан с обычным OB $D(z)$ формулой, аналогичной формуле (3.13). Отсюда следует, что $Ф С С ~ \xi(\lambda)$ непрерывна при $\lambda>0$ и удовлетворяет соотношению (3.14) с остатком $o\left(\lambda^{-1 / 2}\right)$.

Случай $d \geqslant 4$ существенно труднее, поскольку $Ф С С ~ \xi(\lambda)$ выражается только через ОВ для пары $\left(H_{0}+c I\right)^{-m},(H+c I)^{-m}$, не связанный при $m>d / 2-$ $1 \geqslant 1$ прямо с $D_{p}(z)$. Для проверки того, что $\xi-$ непрерывная функция при $\lambda>0$, сравним формулы (2.15) и (3.11) и воспользуемся тем, что функция $\operatorname{Tr}\left(d^{m-2}\left(R_{0}(z)\left(V R_{0}(z)\right)^{k}\right) / d z^{m-2}\right), k \geqslant 2$, непрерывна вплоть до разреза $(0, \infty)$. Отсюда вытекает, что интеграл от $\left(\xi(\lambda)-\xi_{p}(\lambda)\right)(\lambda-z)^{-m}$ по $\lambda$ также является непрерывной функцией от $z$ вплоть до этого разреза. Тогда стандартные результаты об интегралах типа Коши показывают, что функция $\xi(\lambda)-\xi_{p}(\lambda)$ принадлежит классу $C^{m-1}$ для $\lambda>0$. Теперь непрерывность функции $\xi(\lambda)$ следует из теоремы 3.7. Сформулируем этот результат.

Теорема 3.13. Пусть условие (3.2) выполняется при $\rho>d$. Тогда $\Phi C C$ $\xi(\lambda)$ непрерывна при $\lambda>0$.

Используя представление (3.3), легко показать, что в предположении, что $\rho>d, \operatorname{MP} S(\lambda)$ зависит непрерывно от $\lambda>0$ в ядерной топологии, а потому $\operatorname{Det} S(\lambda)$ также является непрерывной функцией от $\lambda>0$. Тем не менее непрерывность $\Phi С С ~ \xi(\lambda)$ не следует из формулы (1.2), так как ее целочисленные скачки заранее не исключены.

Главный член высокоэнергетической асимптотики ФСС дается первым членом в правой части формулы (3.14) для всех $d \geqslant 4$. Этот результат доказывается в $\S 6$ одновременно с полным АР функции $\xi(\lambda)$ при $\lambda \rightarrow \infty$; при этом требуются более ограничительные предположения относительно $v(x)$.

\section{§4. Функция Грина при больших значениях спектрального параметра}

В разд. 1 мы построим АР интегрального ядра $R\left(x, x^{\prime} ; z\right)$ резольвенты (функции Грина) при $|z| \rightarrow \infty$ при условии, что $z \in \Pi_{\theta}$, где $\arg z \in[\theta, 2 \pi-\theta]$ при каком-либо $\theta>0$. Этот метод не работает при $\theta=0$, но зато дает явные выражения для коэффициентов этого разложения и не требует убывания функции $v(x)$ на бесконечности. При некоторых предположениях убывания этот метод дает также AР для $\operatorname{Tr}\left(R^{m}(z)-R_{0}^{m}(z)\right)$. В разд. 2 мы получаем AР параболической 
функции Грина (ядра уравнения теплопроводности) $G\left(x, x^{\prime} ; t\right)$ при $t \rightarrow 0$, что не требует почти никаких предположений о $v(x)$ на бесконечности. Преобразование Лапласа связывает это разложение с АР функции $R\left(x, x^{\prime} ; z\right)$ при $|z| \rightarrow \infty$. В разд. 3 результаты о $G\left(x, x^{\prime} ; t\right)$ используются для усиления результатов о локальном АР функции $R\left(x, x^{\prime} ; z\right)$. Напротив, $\mathrm{AP}$ функции $\operatorname{Tr}\left(R^{m}(z)-R_{0}^{m}(z)\right)$ при $|z| \rightarrow \infty$ используется для вывода АР функции $\operatorname{Tr}\left(e^{-H t}-e^{-H_{0} t}\right)$ при $t \rightarrow 0$.

1. Мы исходим из модификации (см. [13]) проитерированного резольвентного тождества. Ее можно рассматривать как частный случай некоммутативной формулы Тейлора из [16]. Мы приведем это тождество для оператора Шрёдингера, хотя на самом деле оно имеет общий характер.

Предложение 4.1. Предположим, что $v \in C^{\infty}\left(\mathbb{R}^{d}\right)$ u что $v$, a тажже все ее производные являются ограниченными функциями. Определим дифберенциальные выражсения $X_{n}$ рекуррентными формулами

$$
X_{0}=I \quad u \quad X_{n+1}=X_{n} H_{0}-H X_{n},
$$

mak чmo

$$
X_{n}=\sum_{k=0}^{n}(-1)^{k}\left(\begin{array}{l}
n \\
k
\end{array}\right) H^{k} H_{0}^{n-k}
$$

Тогда для всех $N \geqslant 0$

$$
R(z)=\sum_{n=0}^{N} X_{n} R_{0}^{n+1}(z)+R(z) X_{N+1} R_{0}^{N+1}(z) .
$$

Операторы $X_{n}$ можно легко сосчитать. (Ниже $\langle\cdot, \cdot\rangle-$ скалярное произведение в $\mathbb{R}^{d}$.) Например, $X_{1}=-v, X_{2}=-2\langle(\nabla v), \nabla\rangle-(\Delta v)+v^{2}$ и

$$
X_{3}=-4\langle\operatorname{Hess} v \nabla, \nabla\rangle+6 v\langle(\nabla v), \nabla\rangle-\left(\Delta^{2} v\right)+2|\nabla v|^{2}+3 v(\Delta v)-v^{3} .
$$

Предложение 4.2. Оператор $X_{n}$ является дифберенциальным оператором порядка $n-1$, так что

$$
X_{n}=\sum_{|\alpha| \leqslant n-1} p_{\alpha, n} \partial^{\alpha}, \quad p_{\alpha, n}(x)=\overline{p_{\alpha, n}(x)}, \alpha=\left\{\alpha_{1}, \ldots, \alpha_{d}\right\} .
$$

В условиях предложения 4.1 все коэфбииченты $p_{\alpha, n}$, а такэе все их производные суть ограниченные функиии. Кроме того, если v удовлетворяет оценкам

$$
\left\|\partial^{\kappa} v(x)\right\| \leqslant C_{\kappa}(1+\|x\|)^{-\rho-\|\kappa\|}, \quad \rho>0,
$$

для всех мультииндексов $\kappa$, то $\left(\partial^{\kappa} p_{\alpha, n}\right)(x)=O\left(|x|^{-\rho-|\kappa|-(n-1) \epsilon}\right), \epsilon=\min \{\rho, 1\}$, для всех $n, \alpha$ и к при $|x| \rightarrow \infty$. [13]).

Оба предложения 4.1 и 4.2 непосредственно проверяются индукцией по $n$ (см.

Воспользуемся тождеством (4.2) для нахождения АР интегрального ядра $R\left(x, x^{\prime} ; z\right)$ резольвенты $R(z)$ при $|z| \rightarrow \infty, z \in \Pi_{\theta}$. Согласно (4.3), оператор $T_{n}(z)=X_{n} R_{0}^{n+1}(z)$ для $n \geqslant 1$ имеет интегральное ядро

$$
T_{n}\left(x, x^{\prime} ; z\right)=(2 \pi)^{-d} \sum_{|\alpha| \leqslant n-1} i^{|\alpha|} p_{\alpha, n}(x) \int_{\mathbb{R}^{d}} e^{i\left\langle x-x^{\prime}, \xi\right\rangle} \xi^{\alpha}\left(|\xi|^{2}-z\right)^{-n-1} d \xi .
$$


Отсюда следует, что

$$
\left|T_{n}\left(x, x^{\prime} ; z\right)\right| \leqslant C \sum_{|\alpha| \leqslant n-1}\left|p_{\alpha, n}(x)\right||z|^{-(n+3-d) / 2},
$$

если $n+3>d$. Конечно, формула (4.5) может быть переписана в виде

$$
T_{n}\left(x, x^{\prime} ; z\right)=n !^{-1} \sum_{|\alpha| \leqslant n-1} p_{\alpha, n}(x) \partial_{x}^{\alpha} R_{0}^{(n)}\left(x-x^{\prime} ; z\right),
$$

где $R_{0}^{(n)}\left(x-x^{\prime} ; z\right)=\partial_{z}^{n} R_{0}\left(x-x^{\prime} ; z\right)$ и $R_{0}\left(x-x^{\prime} ; z\right)$ - интегральное ядро резольвенты $R_{0}(z)$. Оценка остатка $R(z) X_{N+1} R_{0}^{N+1}(z)$ в (4.2) основана на очевидном соотношении $\|R(z)\|=O\left(|z|^{-1}\right)$ и явной формуле (4.5). Таким образом, тождество (4.2) дает следующий результат.

Теорема 4.3. Предположим, что $v \in C^{\infty}\left(\mathbb{R}^{d}\right)$ u что $v$, a также все ее производные являются ограниченными функииями. Тогда при всех достаточно больших $N(N \geqslant d-3)$ асимптотическое соотношение

$$
R\left(x, x^{\prime} ; z\right)=\sum_{n=0}^{N} T_{n}\left(x, x^{\prime} ; z\right)+O\left(|z|^{-(N-d) / 2-2}\right)
$$

справедливо при $|z| \rightarrow \infty, z \in \Pi_{\theta}$. Оченка остатка здесъ равномерна по $x, x^{\prime} \in$ $\mathbb{R}^{d}$. Кроме того, соотношение (4.7) бесконечно дифберениируемо по $x, x^{\prime}$ ( этом $N$ возрастает) $u z$.

Из равенства (4.6) следует, что функции $T_{n}\left(x, x^{\prime} ; z\right)$ убывают экспоненциально при $\left|x-x^{\prime}\right||z|^{1 / 2} \rightarrow \infty, z \in \Pi_{\theta}$. Поэтому, хотя AР (4.7) и справедливо для всех $x, x^{\prime} \in \mathbb{R}^{d}$, оно представляет интерес только в области $\left|x-x^{\prime}\right|=O\left(|z|^{-1 / 2}\right)$. Заметим, что функции $T_{n}\left(x, x^{\prime} ; z\right)$ сингулярны на диагонали $x=x^{\prime}$, но становятся более гладкими при возрастании $n$. В частности, эти функции непрерывны, если $n>d-3$ и $n \geqslant 1$ (функция $T_{0}\left(x, x^{\prime} ; z\right)$ непрерывна только при $d=1$ ). Таким образом, (4.7) дает разложение функции Грина одновременно при больших $|z|$ и по гладкости по переменной $x-x^{\prime}$. Отметим также, что диагональные сингулярности функций $T_{n}\left(x, x^{\prime} ; z\right)$ ослабевают после дифференцирований по $z$.

Обсудим характер разложения (4.7) при $x \rightarrow x^{\prime}$. Если $d=1$, то можно прямо положить $x=x^{\prime}$ в (4.7). В случае $d \geqslant 2$ мы предварительно продифференцируем $(4.5)$ и $(4.7) m-1$ раз по $z$. Если $2(m+1)>d$, то интегральное ядро оператора $R^{(m-1)}(z)-R_{0}^{(m-1)}(z)$ непрерывно. Поэтому, полагая $x=x^{\prime}$ и собирая вместе члены одной степени по $z$, получим

Следствие 4.4. Пусть $2(m+1)>d$. Положим $c_{\alpha, k}=\int_{\mathbb{R}^{d}} \xi^{2 \alpha}\left(|\xi|^{2}+1\right)^{-k} d \xi$, где $k>|\alpha|+d / 2$. Тогда справедливо $A P$

$$
\left.\left(R^{(m-1)}\left(x, x^{\prime} ; z\right)-R_{0}^{(m-1)}\left(x, x^{\prime} ; z\right)\right)\right|_{x=x^{\prime}}=(m-1) ! \sum_{n=0}^{\infty} r_{n}^{(m)}(x)(-z)^{d / 2-m-n-1}
$$

где (это определение имеет смысл при $n+m+1>d / 2$ )

$$
r_{n}^{(m)}(x)=(2 \pi)^{-d} \sum_{|\alpha| \leqslant n}(-1)^{|\alpha|}\left(\begin{array}{c}
|\alpha|+n+m \\
m-1
\end{array}\right) c_{\alpha,|\alpha|+n+m+1} p_{2 \alpha, n+|\alpha|+1}(x) .
$$


Для потенциалов $v$, убывающих на бесконечности, AР (4.8) может быть проинтегрировано по $x \in \mathbb{R}^{d}$, потому что производная порядка $m-1$ остатка в (4.2) оценивается в ядерной норме. Это дает следующий результат.

Теорема 4.5. Пусть предположение (4.4) выполняется при $\rho>d$, , пусть $2(m+1)>d$. Тогда при $|z| \rightarrow \infty, z \in \Pi_{\theta}$, справедливо АР

$$
\operatorname{Tr}\left(R^{m}(z)-R_{0}^{m}(z)\right)=\sum_{n=0}^{\infty} \mathbf{r}_{n}^{(m)}(-z)^{d / 2-m-n-1} .
$$

Вещественные коэффициенты $\mathbf{r}_{n}^{(m)}$ определяются формулой

$$
\mathbf{r}_{n}^{(m)}=\int_{\mathbb{R}^{d}} r_{n}^{(m)}(x) d x .
$$

Замечание 4.6. Вместо дифференцирований можно вычитать из $R(z)$ несколько (а не один, как в (4.10)) членов разложения (4.2). Это позволяет ослабить условие $\rho>d$. Например, если $d<6$ и (4.4) справедливо для $\rho>d / 2$, то

$$
\operatorname{Tr}\left(R(z)-R_{0}(z)+R_{0}(z) V R_{0}(z)\right)=\sum_{n=1}^{\infty} \mathbf{r}_{n}^{(1)}(-z)^{d / 2-n-2} .
$$

2. Параболическая функция Грина $G\left(x, x^{\prime} ; t\right)$ определяется как интегральное ядро оператора $\exp (-H t), t>0$. Она удовлетворяет параболическому уравнению

$$
\partial G\left(x, x^{\prime} ; t\right) / \partial t=\Delta G\left(x, x^{\prime} ; t\right)-v(x) G\left(x, x^{\prime} ; t\right), \quad \Delta=\Delta_{x} .
$$

Мы ищем (ср. с [2]) его приближенное решение в виде

$$
G_{N}\left(x, x^{\prime} ; t\right)=G_{0}\left(x, x^{\prime} ; t\right) \sum_{n=0}^{N} g_{n}\left(x, x^{\prime}\right) t^{n},
$$

где $G_{0}\left(x, x^{\prime} ; t\right)=G_{0}\left(x-x^{\prime} ; t\right)$ - интегральное ядро оператора $\exp \left(-H_{0} t\right)$ и $g_{0}\left(x, x^{\prime}\right)=1$. Подставим выражение (4.14) в (4.13), воспользуемся уравнением $\partial G_{0} / \partial t=\Delta G_{0}$ и разделим на общий множитель $G_{0}$. Равенство нулю коэффициентов при $t^{n}, n=0,1, \ldots, N-1$, приводит к рекуррентным уравнениям для функций $g_{n+1}\left(x, x^{\prime}\right)$. Для фиксированного $x^{\prime}$ это дает обыкновенное дифференциальное уравнение $(n+1) g_{n+1}+r \partial g_{n+1} / \partial r=\Delta g_{n}-v g_{n}$ по переменной $r=\left|x-x^{\prime}\right|$. Решая его при условии, что величина $g_{n+1}(x, x)$ конечна, получим формулу

$$
g_{n+1}\left(x, x^{\prime}\right)=\int_{0}^{1} \sigma^{n}\left(\Delta g_{n}-v g_{n}\right)\left(x^{\prime}+\sigma\left(x-x^{\prime}\right), x^{\prime}\right) d \sigma .
$$

Приведенная конструкция не требует никаких предположений о поведении $v(x)$ на бесконечности. Обоснование локального АР функции $G\left(x, x^{\prime} ; t\right)$ при $t \rightarrow 0$ проходит при очень слабых предположениях. Вначале, пользуясь равенством (4.15), проверяем по индукции следующее утверждение.

Лемма 4.7. Предположим, ито $v \in C^{\infty}\left(\mathbb{R}^{d}\right)$ u $\left(\partial^{\kappa} v\right)(x)=O\left(e^{\gamma|x|^{2}}\right) n p u$ $|x| \rightarrow \infty$ для некоторого $\gamma \geqslant 0$ и всех $\kappa$. Тогда $\left|g_{n}\left(x, x^{\prime}\right)\right| \leqslant C_{n} e^{(n \gamma+\varepsilon)\left|x-x^{\prime}\right|^{2}} \partial_{\Omega \varkappa}$ всех $\varepsilon>0$ равномерно по $x^{\prime}$ из компактных подмножеств пространства $\mathbb{R}^{d}$. Кроме того, эти оценки бесконечно дифберениируемы по переменным $x$ u $x^{\prime}$. 
Положим $Q_{N}=G_{0}\left(-\Delta g_{N}+v g_{N}\right) t^{N}$. Из (4.13) и аналогичного уравнения для функции $G_{N}$ следует, что разность $F_{N}=G-G_{N}$ допускает представление

$$
F_{N}\left(x, x^{\prime} ; t\right)=-\int_{0}^{t}\left(e^{-(t-s) H} Q_{N}\left(\cdot, x^{\prime} ; s\right)\right)(x) d s,
$$

где $x^{\prime}$ фиксировано. В формуле (4.16), конечно, подразумевается, что оператор $e^{-(t-s) H}$ действует на функцию $Q_{N}\left(x, x^{\prime} ; s\right)$ по первой переменной, а $x^{\prime}$ и $s$ играют роль параметров. Комбинируя оценку из леммы 4.7 для $g_{N}$ с очевидным неравенством $\left\|e^{-t H}\right\| \leqslant e^{-\lambda_{1} t}$, можно оценить функцию (4.16) для малых $t$ через $C_{N} t^{N-d / 4+1}$ равномерно по $x, x^{\prime}$ из компактных множеств. Это дает следующий результат.

Теорема 4.8. Пусть выполнены условия леммы 4.7. Предположим, что оператор $H=-\Delta+v(x)$ самосопрлжсен на области определения $\mathscr{D}(H) \supset$ $\mathscr{S}\left(\mathbb{R}^{d}\right)$ и что он полуограничен снизу. Определим функиии $g_{n}$ рекуррентными соотношениями $g_{0}\left(x, x^{\prime}\right)=1$ u (4.15). Тогда $A P$

$$
G\left(x, x^{\prime} ; t\right)=(4 \pi t)^{-d / 2} \exp \left(-(4 t)^{-1}\left|x-x^{\prime}\right|^{2}\right) \sum_{n=0}^{\infty} g_{n}\left(x, x^{\prime}\right) t^{n}
$$

справедливо при $t \rightarrow 0$ равномерно по $x$ u $x^{\prime}$ из компактных подмножеств пространства $\mathbb{R}^{d}$. В частности,

$$
G(x, x ; t)=(4 \pi t)^{-d / 2} \sum_{n=0}^{\infty} g_{n}(x) t^{n}, \quad g_{n}(x)=g_{n}(x, x), t \rightarrow 0 .
$$

Кроме того, АР (4.17) и (4.18) бесконечно дифференцируемы по переменным $x, x^{\prime} u t$.

Конечно, соотношение (4.17) содержательно только при $\left|x-x^{\prime}\right|=O\left(t^{1 / 2}\right)$.

Исходя из (4.15), можно дать явные выражения для функций $g_{n}\left(x, x^{\prime}\right)$. В частности, нетрудно сосчитать первые функции $g_{n}(x)$ (называемые локальными инвариантами уравнения теплопроводности для оператора $H)$ :

$$
\begin{gathered}
g_{1}=-v, \quad g_{2}=2^{-1} v^{2}-6^{-1} \Delta v, \quad g_{3}=-6^{-1}\left(v^{3}-v \Delta v-2^{-1}|\nabla v|^{2}+10^{-1} \Delta^{2} v\right), \\
g_{4}=24^{-1} v^{4}+30^{-1}\langle\nabla v, \nabla(\Delta v)\rangle+60^{-1} v \Delta^{2} v+72^{-1}(\Delta v)^{2}-840^{-1} \Delta^{3} v \\
-12^{-1} v^{2} \Delta v-12^{-1} v|\nabla v|^{2}+90^{-1} \operatorname{Tr}(\operatorname{Hess} v)^{2} .
\end{gathered}
$$

Для произвольного $n$ функции $g_{n}(x)$ выражаются (см. [26], а также [14], где использовались результаты из [1]) формулой

$$
g_{n}(x)=(-1)^{n} \Gamma(n+d / 2) \sum_{k=0}^{n-1} \frac{\left.\left(-\Delta_{x}+v(x)\right)^{k+n}\left(\left|x-x^{\prime}\right|^{2 k}\right)\right|_{x=x^{\prime}}}{4^{k} k !(k+n) !(n-1-k) ! \Gamma(k+d / 2+1)} .
$$

3. Поскольку

$$
R\left(x, x^{\prime} ; z\right)=\int_{0}^{\infty} G\left(x, x^{\prime} ; t\right) e^{t z} d t, \quad \operatorname{Re} z<\inf \sigma, \sigma=\mathbb{C} \backslash \varrho,
$$

разложение (4.17) при $t \rightarrow 0$ можно связать с асимптотическим разложением ядра резольвенты $R\left(x, x^{\prime} ; z\right)$ при $|z| \rightarrow \infty$ и тем самым усилить результаты 
разд. 1. Подчеркнем, что ограниченность функции $v(x)$ сейчас не предполагается.

Теорема 4.9. В условиях теоремы 4.8 для достаточно больиих $N$

$$
R\left(x, x^{\prime} ; z\right)=\sum_{n=0}^{N} g_{n}\left(x, x^{\prime}\right) R_{0}^{(n)}\left(x, x^{\prime} ; z\right)+O\left(|z|^{-N+d / 2-2}\right)
$$

при $|z| \rightarrow \infty, \operatorname{Re} z<\inf \sigma$, равномерно по $x$ u $x^{\prime}$ из компактных подмножеств пространства $\mathbb{R}^{d}$. Разложение (4.21) бесконечно дифберениируемо по $x, x^{\prime}$ $u z$.

Теоремы 4.3 и 4.9 дают два различных разложения ядра резольвенты. Аналогично тому, как это делалось в разд. 1, после дифференцирований по $z$ в (4.21) можно перейти к пределу при $x^{\prime} \rightarrow x$. Напомним, что ядро $R_{0}\left(x, x^{\prime} ; z\right)$ сингулярно на диагонали, но его производные $R_{0}^{(n)}\left(x, x^{\prime} ; z\right)$ становятся более гладкими при возрастании $n$. В частности, функция $R_{0}^{(n)}\left(x, x^{\prime} ; z\right)$ непрерывна на диагонали, если $2(n+1)>d$, и

$$
R_{0}^{(n)}(x, x ; z)=(4 \pi)^{-d / 2} \Gamma(1+n-d / 2)(-z)^{d / 2-n-1} .
$$

Поэтому, вычитая из (4.21) функцию $R_{0}\left(x, x^{\prime} ; z\right)$ и дифференцируя $m-1$ раз по $z$, можно при $2(m+1)>d$ положить $x=x^{\prime}$. Это дает АР

$$
(4 \pi)^{-d / 2} \sum_{n=0}^{\infty} \Gamma(n+m+1-d / 2) g_{n+1}(x)(-z)^{d / 2-m-n-1}
$$

для левой части формулы (4.8). Сравнивая его с правой частью этой формулы, получаем, что

$$
r_{n}^{(m)}(x)=(4 \pi)^{-d / 2}(m-1) !^{-1} \Gamma(n+m+1-d / 2) g_{n+1}(x) .
$$

Интегрируя (4.18) по $x \in \mathbb{R}^{d}$, мы формально получим АР

$$
\operatorname{Tr}\left(e^{-H t}-e^{-H_{0} t}\right)=(4 \pi t)^{-d / 2} \sum_{n=1}^{\infty} \mathbf{g}_{n} t^{n}, \quad \text { где } \mathbf{g}_{n}=\int_{\mathbb{R}^{d}} g_{n}(x) d x .
$$

Переход от $(4.18)$ к (4.23) требует некоторых оценок функции $F_{N}(x, x ; t)$ на бесконечности, что, в свою очередь, требует подходящего убывания потенциала при $|x| \rightarrow \infty$. Мы избегаем таких оценок и выводим AР (4.23) из теоремы 4.5, используя формулу (обращение преобразования Лапласа (4.20))

$$
\operatorname{Tr}\left(e^{-H t}-e^{-H_{0} t}\right)=(2 \pi i)^{-1}(m-1) ! t^{-m+1} \int_{\beta-i \infty}^{\beta+i \infty} \operatorname{Tr}\left(R^{m}(z)-R_{0}^{m}(z)\right) e^{-t z} d z,
$$

где $2(m+1)>d$ и $\beta<\inf \sigma$. Сформулируем точный результат.

Теорема 4.10. Предположим, что $v(x)$ удовлетворяет оценкам (4.4), где $\rho>d$. Тогда справедливо АP (4.23).

Наконец, отметим, что, согласно (4.22),

$$
\mathbf{r}_{n}^{(m)}=(4 \pi)^{-d / 2}(m-1) !^{-1} \Gamma(n+m+1-d / 2) \mathbf{g}_{n+1} .
$$




\section{§5. Высокоэнергетическая асимптотика MP}

1. Пусть выполнено условие (4.4). Вне диагонали $\omega=\omega^{\prime}$ интегральное ядро $s\left(\omega, \omega^{\prime} ; \lambda\right) \mathrm{MP} S(\lambda)$ является $C^{\infty}$-функцией и убывает быстрее любой степени $\lambda^{-1}$ при $\lambda \rightarrow \infty$. Напротив, оно сингулярно на диагонали, причем его сингулярности определяются убыванием функции $v(x)$ на бесконечности. Оказывается, что эти сингулярности и высокоэнергетический предел описываются общими формулами.

Для описания $s\left(\omega, \omega^{\prime} ; \lambda\right)$ в окрестности диагонали напомним стандартную конструкцию приближенных, но зато явных решений $\psi_{N}$ уравнения Шрёдингера $-\Delta \psi+v(x) \psi=\lambda \psi$. Эта конструкция основана на решении итерациями соответствующего уравнения переноса. Считая $\rho>1$, положим

$$
\psi_{N}(x, \xi)=e^{i\langle x, \xi\rangle} \mathrm{b}_{N}(x, \xi), \quad \xi=k \omega \in \mathbb{R}^{d}, k=\sqrt{\lambda},
$$

где

$$
\mathrm{b}_{N}(x, \xi)=\sum_{n=0}^{N}(2 i k)^{-n} b_{n}(x, \omega), \quad b_{0}(x, \omega)=1 .
$$

Подставляя эти выражения в уравнение Шрёдингера и приравнивая коэффициенты при одинаковых степенях $(2 i k)^{-1}$, получим рекуррентные уравнения

$$
\left\langle\omega, \nabla_{x} b_{n+1}(x, \omega)\right\rangle=-\Delta b_{n}(x, \omega)+v(x) b_{n}(x, \omega),
$$

откуда следует, что

$$
b_{n+1}(x, \omega)=\int_{-\infty}^{0}\left(-\Delta b_{n}(x+t \omega, \omega)+v(x+t \omega) b_{n}(x+t \omega, \omega)\right) d t .
$$

Легко показать, что при условии (4.4) для любого $c<1$ и $\varepsilon_{0}=\min \{1, \rho-1\}$

$$
\left|\partial_{x}^{\alpha} b_{n}(x, \omega)\right| \leqslant C_{\alpha}(1+|x|)^{-\rho+1-\varepsilon_{0}(n-1)-|\alpha|}, \quad n \geqslant 1, \quad \text { если }\langle x, \omega\rangle \leqslant c|x| .
$$

Фиксируем какую-либо точку $\omega_{0} \in \mathbb{S}^{d-1}$. Пусть $\Lambda\left(\omega_{0}\right)-$ плоскость, ортогональная $\omega_{0}$, и $\Omega=\Omega\left(\omega_{0}, \delta\right) \subset \mathbb{S}^{d-1}$ определяется условием $\left\langle\omega, \omega_{0}\right\rangle>\delta>0$. Положим $x=\omega_{0} z+y$, где $y \in \Lambda\left(\omega_{0}\right)$,

$$
\begin{aligned}
& A_{N}\left(\omega, \omega^{\prime}, y ; \lambda\right)=2^{-1}\left\langle\omega+\omega^{\prime}, \omega_{0}\right\rangle \mathrm{b}_{N}(y,-k \omega) \mathrm{b}_{N}\left(y, k \omega^{\prime}\right) \\
& \quad+(2 i k)^{-1}\left(\mathrm{~b}_{N}(y,-k \omega)\left(\partial_{z} \mathrm{~b}_{N}\right)\left(y, k \omega^{\prime}\right)-\mathrm{b}_{N}\left(y, k \omega^{\prime}\right)\left(\partial_{z} \mathrm{~b}_{N}\right)(y,-k \omega)\right)
\end{aligned}
$$

и

$$
s_{N}\left(\omega, \omega^{\prime} ; \lambda\right)=(2 \pi)^{-d+1} k^{d-1} \int_{\Lambda_{\omega_{0}}} e^{i k\left\langle y, \omega^{\prime}-\omega\right\rangle} A_{N}\left(\omega, \omega^{\prime}, y ; \lambda\right) d y
$$

для $\omega, \omega^{\prime} \in \Omega$. Поскольку $|\langle y, \omega\rangle| \leqslant c|y|$ и $\left|\left\langle y, \omega^{\prime}\right\rangle\right| \leqslant c|y|$, где $c<1$, для $\omega, \omega^{\prime} \in \Omega$, $y \in \Lambda\left(\omega_{0}\right)$, оценки (5.3) гарантируют, что осциллирующий интеграл (5.5) корректно определен. Как показано в [34], функция $s_{N}\left(\omega, \omega^{\prime} ; \lambda\right)$ описывает все сингулярности функции $s\left(\omega, \omega^{\prime} ; \lambda\right)$ и аппроксимирует ее с произвольной точностью при $\lambda \rightarrow \infty$. А именно, справедлива

Теорема 5.1. Пусть условие (4.4) выполнено при $\rho>1$, а функиия $\mathrm{b}_{N}(x, \xi)$ определяется формулами (5.1) u (5.2). При $\omega, \omega^{\prime} \in \Omega$ определим функиию $s_{N}$ равенствами (5.4), (5.5). Тогда при любом $p$ и достаточно большом $N=N(p)$ остаток $s\left(\omega, \omega^{\prime} ; \lambda\right)-s_{N}\left(\omega, \omega^{\prime} ; \lambda\right)$ принадлежит классу $C^{p}(\Omega \times \Omega)$ и $C^{p}$-норма этой функиии есть $O\left(\lambda^{-p}\right)$ при $\lambda \rightarrow \infty$. 
Теорема 5.1 распространяется на медленно убывающие потенциалы (см. [35]).

Отметим, что другая форма АР амплитуды рассеяния $s\left(\omega, \omega^{\prime}, \lambda\right)$ содержится в [31].

Формула (5.5) показывает, что по существу мы рассматриваем MP $S(\lambda)$ как псевдодифференциальный оператор на единичной сфере, задаваемый его амплитудой $A\left(\omega, \omega^{\prime}, y ; \lambda\right)$. Подставляя выражение (5.1) в правую часть формулы (5.4), можно разложить эту амплитуду в асимптотический ряд по степеням $(2 i k)^{-1}$. Кроме того, теорему 5.1 можно переформулировать, используя стандартную процедуру (см, например, [30]) перехода от амплитуды псевдодифференциального оператора к его символу. Отметим, что операторная функция $S$ лежит в $C^{\infty}\left(\mathbb{R}_{+}\right)$, и ее $\mathrm{AP}$ при $\lambda \rightarrow \infty$ бесконечно дифференцируемо по $\lambda$.

2. Теперь мы рассмотрим оператор $T(\lambda)=-i S^{*}(\lambda) S^{\prime}(\lambda)$, называемый оператором временно́й задержки Эйзенбуда-Вигнера. Ввиду унитарности МР оператор $T(\lambda)$ самосопряжен. Заметим, что его ядро $t\left(\omega, \omega^{\prime} ; \lambda\right)$ имеет порядок $O\left(\lambda^{-\infty}\right)$ при $\omega \neq \omega^{\prime}$. Стандартные результаты псевдодифференциального исчисления (см, например, [30]) показывают, что, наряду с $S(\lambda)$ и $S^{\prime}(\lambda)$, оператор $T(\lambda)$ также является псевдодифференциальным. Кроме того, из теоремы 5.1 вытекает следующий результат об операторе $T(\lambda)$, который мы сформулируем в терминах его правого символа.

Теорема 5.2. Пусть условие (4.4) выполняется при $\rho>1$. Тогда ядро оператора $T(\lambda)$ временной задержки допускает AP

$$
t\left(\omega, \omega^{\prime} ; \lambda\right)=(2 \pi)^{-d+1} k^{d-4} \sum_{n=0}^{\infty}(2 i k)^{-n} \int_{\Lambda_{\omega}} e^{i k\left\langle y, \omega^{\prime}-\omega\right\rangle} \mathrm{t}_{n}\left(\omega^{\prime}, y\right) d y,
$$

где $\mathrm{t}_{n}=\overline{\mathrm{t}}_{n}$ суть гладкие функции переменных $\omega^{\prime} \in \Omega(\omega, \delta)$ и у $\in \Lambda(\omega), a$ $\partial_{y}^{\alpha} \mathrm{t}_{n}\left(\omega^{\prime}, y\right)=O\left(|y|^{-\rho+1-\varepsilon_{0} n-|\alpha|}\right)$ nри $|y| \rightarrow \infty$. Кроме того, $T \in C^{\infty}\left(\mathbb{R}_{+}\right) u$ $A P$ (5.6) можно бесконечно дифберениировать по $\lambda$. Если $\rho>d$, то ядро $t-$ непрерывная функиия переменных $\omega, \omega^{\prime} u$

$$
t(\omega, \omega ; \lambda)=(2 \pi)^{-d+1} \lambda^{d / 2-2} \sum_{n=0}^{\infty}(-4 \lambda)^{-n} \tau_{n}(\omega),
$$

где $\tau_{n}(\omega)$ - интегралы от $\mathrm{t}_{2 n}(\omega, y)$ по $y \in \Lambda(\omega)$. АР (5.7) такље бесконечно дифферениируемо по $\lambda$.

\section{§6. Высокоэнергетическая асимптотика ФСС}

Наша цель в этом параграфе состоит в получении полного асимптотического разложения $Ф \mathrm{CC} \xi(\lambda)$ при $\lambda \rightarrow \infty$. В то же время мы распространяем AР (4.10) на всю комплексную плоскость $\Pi_{0}$ с разрезом по положительной полуоси (включая разрез). В разд. 1 мы устанавливаем существование полного $\mathrm{AP} \xi(\lambda)$. Однако используемый там метод дает сложные выражения для коэффициентов $\xi_{n}$ этого разложения. Этот недостаток исправляется в разд. 2 и 3 , где находится связь результатов разд. 1 с результатами разд. 1 и $2 \S 4$. Это дает выражения для $\xi_{n}$ в терминах коэффициентов $\mathbf{r}_{n}^{(m)}$, а также инвариантов $\mathbf{g}_{n+1}$ уравнения теплопроводности.

1. Будем исходить из уравнения (2.9), которое следует из формулы БирманаКрейна (1.2), поскольку ФСС $\xi-$ непрерывная функция переменной $\lambda>0$ и 
операторная функция $S(\lambda)$ дифференцируема по $\lambda$ в ядерной норме. Затем мы пользуемся теоремой 5.2. Интегрируя (5.7) по $\omega \in \mathbb{S}^{d-1}$ и учитывая формулу $(2.9)$, получаем АР производной $\xi^{\prime}(\lambda)$ ФСС $\xi$.

Теорема 6.1. Пусть условие (4.4) выполняется для $\rho>d$. Тогда $\xi \in$ $C^{\infty}\left(\mathbb{R}_{+}\right)$и справедливо АР

$$
\xi^{\prime}(\lambda)=\lambda^{d / 2-2} \sum_{n=0}^{\infty} \eta_{n} \lambda^{-n}
$$

с коэффиииентами

$\eta_{0}=4^{-1}(d-2)(2 \pi)^{-d}\left|\mathbb{S}^{d-1}\right| \int_{\mathbb{R}^{d}} v(x) d x, \quad \eta_{n}=-(2 \pi)^{-d}(-4)^{-n} \int_{\mathbb{S}^{d-1}} \tau_{n}(\omega) d \omega$.

Разложение (6.1) можно бесконечно дифферениировать.

Интегрируя (6.1) по $\lambda$, получаем следующий результат.

Следствие 6.2. Для подходящей постоянной $\gamma \Phi C C \xi(\lambda)$ допускает $A P$

$$
\xi(\lambda)=\lambda^{d / 2-1} \sum_{n=0, n \neq d / 2-1}^{\infty} \xi_{n} \lambda^{-n}+\tilde{\xi} \ln \lambda+\gamma,
$$

где $\xi_{n}=(d / 2-n-1)^{-1} \eta_{n}, \tilde{\xi}=0$ при нечетном $d$ и $\tilde{\xi}=\eta_{d / 2-1}$ при четном $d$.

Выражения (6.2) для коэффициентов $\eta_{n}, n \geqslant 1$, довольно сложны. Ниже мы найдем эффективные выражения для $\xi_{n}$. Одновременно мы покажем, что $\tilde{\xi}=0$ для всех $d$ и найдем постоянную $\gamma$ в (6.3).

2. Ввиду представления (2.15) следующее утверждение может быть выведено из следствия 6.2 с помощью варианта теоремы Привалова.

Предложение 6.3. Пусть условие (4.4) выполняется для $\rho>d, u$ пусть $2(m+1)>d$. Тогда для некоторых чисел $c_{n}^{(m)}, n \geqslant m+1$, при $|z| \rightarrow \infty, z \in \Pi_{0}$, имеют место $A P$

$$
\operatorname{Tr}\left(R^{m}(z)-R_{0}^{m}(z)\right)=\sum_{n=0}^{\infty} \mathbf{r}_{n}^{(m)}(-z)^{d / 2-m-n-1}-\gamma(-z)^{-m}+\sum_{n=m+1}^{\infty} c_{n}^{(m)} z^{-n},
$$

если d нечетно, и

$$
\begin{aligned}
\operatorname{Tr}\left(R^{m}(z)-R_{0}^{m}(z)\right) & =\sum_{n=0}^{d / 2-2} \mathbf{r}_{n}^{(m)}(-z)^{d / 2-m-n-1} \\
& +\sum_{n=d / 2}^{\infty} \sigma_{n}^{(m)}(-z)^{d / 2-m-n-1} \ln (-z)-\gamma(-z)^{-m}+\sum_{n=m+1}^{\infty} c_{n}^{(m)} z^{-n},
\end{aligned}
$$

если d четно (сумма по $n=0, \ldots, d / 2-2$ отсутствует, если $d=2$ ). Здесь $\gamma$ то же самое, что в (6.3), а асимптотические коэффициенты $\mathbf{r}_{n}^{(m)}$ определяются формулой

$$
\mathbf{r}_{n}^{(m)}=-m \mathrm{~B}(-n+d / 2, m+n+1-d / 2) \xi_{n}
$$


$(\mathrm{B}(p, q)$ - бета-функиия) для всех $n$, если d нечетно, и для $n=0, \ldots, d / 2-2$, если d четно, и $\sigma_{n}^{(m)}=-(-1)^{n-d / 2} m(\underset{m}{n-d / 2+m}) \xi_{n}$ для $n=d / 2, d / 2+1, \ldots$.

Подчеркнем, что коэффициенты $c_{n}^{(m)}$ не определяются асимптотическим разложением ФСС $\xi(\lambda)$ при $\lambda \rightarrow \infty$. Сравним предложение 6.3 с теоремой 4.5. Разложение (4.10) должно совпадать с (6.4) или (6.5) в секторе $z \in \Pi_{\theta}$, где $\theta>0$. Поэтому коэффициенты (4.11) и (6.6) также совпадают. Кроме того, $\gamma=0, c_{n}^{(m)}=0$ в (6.4) и $\gamma=-\mathbf{r}_{d / 2-1}^{(m)}, \sigma_{n}^{(m)}=0, c_{n}^{(m)}=\mathbf{r}_{n+d / 2-1}^{(m)}$ в (6.5). Таким образом, из предложения 6.3 вытекает следующий результат.

Теорема 6.4. Пусть условие (4.4) выполняется для $\rho>d$, и пусть $2(m+1)$ $>d$. Тогда AP (4.10) при $|z| \rightarrow \infty$ справедливо при всех $z \in \Pi_{0}$.

В то же время мы существенно улучшили теорему 6.1 и следствие 6.2 , получив явные выражения для коэффициентов $\xi_{n}$ в разложении (6.3) в терминах $\mathbf{r}_{n}^{(m)}$. А именно, справедлива

Теорема 6.5. Пусть условие (4.4) выполняется для $\rho>d$. Тогда ФСС $\xi(\lambda)$ допускает $A P$

$$
\xi(\lambda)=\lambda^{d / 2-1} \sum_{n=0}^{\infty} \xi_{n} \lambda^{-n}
$$

где коэффичиенты $\xi_{n}$ определяются формулами (6.6) и (4.9), (4.11). В частности, $\xi_{n}=0$ для всех $n \geqslant d / 2$, если $d$ четно.

3. AP (6.7) может быть также выведено из теоремы 4.10 (вместо теоремы 4.5). Поскольку

$$
\operatorname{Tr}\left(e^{-H t}-e^{-H_{0} t}\right)=-t \int_{-\infty}^{\infty} \xi(\lambda) e^{-t \lambda} d \lambda,
$$

из АР (6.3) следует (см, например, [10]), что при $t \rightarrow 0$

$$
\operatorname{Tr}\left(e^{-H t}-e^{-H_{0} t}\right)=-\sum_{n=0}^{\infty} \Gamma(d / 2-n) \xi_{n} t^{n+1-d / 2}-\pi \gamma+\sum_{n=1}^{\infty}(-1)^{n} c_{n} t^{n},
$$

если $d$ нечетно, и

$$
\begin{aligned}
\operatorname{Tr}\left(e^{-H t}-\right. & \left.e^{-H_{0} t}\right)=-\sum_{n=0}^{d / 2-1} \Gamma(d / 2-n) \xi_{n} t^{n+1-d / 2}-\pi \gamma \\
& -\sum_{n=d / 2}^{\infty}(-1)^{n-d / 2}((n-d / 2) !)^{-1} \xi_{n} t^{n+1-d / 2} \ln t+\sum_{n=1}^{\infty}(-1)^{n} c_{n} t^{n},
\end{aligned}
$$

если $d$ четно. Здесь

$c_{n}=(n-1) !^{-1}\left(\int_{-\infty}^{0} \xi(\lambda) \lambda^{n-1} d \lambda+\int_{0}^{\infty}\left(\xi(\lambda)-\sum_{j<n+d / 2-1} \xi_{j} \lambda^{d / 2-j-1}\right) \lambda^{n-1} d \lambda\right)$.

Сравнивая (6.8) и (6.9) с (4.23), мы вновь приходим к теореме 6.5. Кроме того, мы получаем явные выражения

$$
\xi_{n}=-(4 \pi)^{-d / 2} \Gamma^{-1}(d / 2-n) \mathbf{g}_{n+1}, \quad n=0,1, \ldots,
$$


для асимптотических коэффициентов в (6.7) в терминах инвариантов уравнения теплопроводности, определяемых формулами (4.19) и (4.23). Соотношение (6.11) может быть также получено сопоставлением формул (4.24) и (6.6).

\section{§7. Тождества следов}

Вклад непрерывного спектра в тождества следов, грубо говоря, определяется функцией $\operatorname{Tr}\left(R(\lambda+i 0)-R_{0}(\lambda+i 0)\right)$. В случае тождеств целого порядка рассматривается мнимая часть этой функции, а в случае тождеств полуцелого порядка - ее вещественная часть.

1. Начнем с формул целого порядка. Применяемый здесь метод не требует никакого специального исследования ОВ или ФСС в окрестности точки $\lambda=0$. В частности, в случае $d=1$ теорема 7.1 не исключает, что оператор $H$ имеет бесконечное число отрицательных собственных значений.

Сравним коэффициент при $t^{n}, n=1,2, \ldots$, в разложении $(4.23)$ с тем же коэффициентом в разложениях (6.8) или (6.9). Согласно (4.23), этот коэффициент равен нулю, если $d$ нечетно, и $(4 \pi)^{-d / 2} \mathbf{g}_{d / 2+n}$, если $d$ четно. С другой стороны, согласно (6.8) или (6.9), он равен $(-1)^{n} c_{n}$ и, следовательно, определяется равенством (6.10). Учтем также, что для $\lambda<0$ ФСС дается формулой (3.5), откуда следует равенство

$$
\int_{-\infty}^{0} \xi(\lambda) \lambda^{n-1} d \lambda=n^{-1} \sum_{j=1}^{\mathrm{N}} \lambda_{j}^{n}
$$

Таким образом, равенства $c_{n}=0$ для нечетных $d$ и $c_{n}=(-1)^{n}(4 \pi)^{-d / 2} \mathbf{g}_{d / 2+n}$ для четных $d$ приводят к тождествам, называемым тождествами следов.

Теорема 7.1. Пусть условие (4.4) выполняется для $\rho>d$. Тогда для всех $n=1,2, \ldots$ справедливы следующие тождества (әде коэффициенты $\xi_{j}$ те же, что в теореме 6.5):

$$
\int_{0}^{\infty}\left(\xi(\lambda)-\sum_{j=0}^{(d-3) / 2+n} \xi_{j} \lambda^{d / 2-j-1}\right) \lambda^{n-1} d \lambda+n^{-1} \sum_{j=1}^{\mathrm{N}} \lambda_{j}^{n}=0,
$$

если $d$ нечетно, и

$\int_{0}^{\infty}\left(\xi(\lambda)-\sum_{j=0}^{d / 2-1} \xi_{j} \lambda^{d / 2-j-1}\right) \lambda^{n-1} d \lambda+n^{-1} \sum_{j=1}^{\mathrm{N}} \lambda_{j}^{n}=(-1)^{n}(4 \pi)^{-d / 2}(n-1) ! \mathbf{g}_{d / 2+n}$,

если $d$ четно.

Формулы (7.1) были установлены в работах [9] и [8] соответственно для случаев $d=1$ и $d=3$. Метод этих работ существенно отличается от предлагаемого здесь, а условия на $v(x)$ значительно более ограничительны. Еще один подход к выводу тождеств типа (7.1) и (7.2) можно найти в [29].

2. Теперь мы получим тождества следов полуцелого порядка. Будем предполагать, что $d \leqslant 3$; это позволит сформулировать результаты в терминах регуляризованного ОВ $D_{2}(z)$, обсуждавшегося в $\S 3$ (при $d=1$ результаты могут быть сформулированы в терминах $D(z)$, см. [12]). 
Предложение 7.2. Пусть $d \leqslant 3$, и пусть условие (4.4) выполняется для $\rho>d / 2$. Тогда имеет место $A P$

$$
\ln D_{2}(z)=\sum_{n=1}^{\infty} \delta_{n}(-z)^{d / 2-1-n}
$$

при $|z| \rightarrow \infty, z \in \Pi_{0}$, где $\delta_{n}=-(n+1-d / 2)^{-1} \mathbf{r}_{n}^{(1)}$, а коэффициенты $\mathbf{r}_{n}^{(1)}$ определяются формулами (4.9) и (4.11). В частности, при $\lambda \rightarrow \infty$ имеем $\ln \left|D_{2}(\lambda \pm i 0)\right|=O\left(\lambda^{-\infty}\right)$, если d нечетно, и

$$
\ln \left|D_{2}(\lambda \pm i 0)\right|=(-1)^{d / 2+1} \sum_{n=1}^{\infty}(-1)^{n} \delta_{n} \lambda^{d / 2-1-n},
$$

если d четно.

Действительно, воспользуемся замечанием 4.6 к теореме 4.5. Сопоставляя уравнение (2.19) для $p=2$ с АР (4.12), мы видим, что

$$
\frac{d \ln D_{2}(z)}{d z}=-\sum_{n=1}^{\infty} \mathbf{r}_{n}^{(1)}(-z)^{d / 2-2-n}
$$

Остается проинтегрировать это АР и учесть соотношение (3.6).

В силу (4.24), кроме того, $\delta_{n}=-(4 \pi)^{-d / 2} \Gamma(n+1-d / 2) \mathbf{g}_{n+1}$.

Теорема 7.3. Пусть $d \leqslant 3$, условие (4.4) выполняется для $\rho>2$ и $n=$ $0,1,2, \ldots$ Предположим, что соотношение (3.8) справедливо при $\alpha=n+1 / 2$. Пусть числа $\delta_{n}$ те же, что в предложсении 7.2. Тогда для $d=1,3$

$$
\pi^{-1}(-1)^{n} \int_{0}^{\infty} \ln \left|D_{2}(\lambda+i 0)\right| \lambda^{n-1 / 2} d \lambda-(n+1 / 2)^{-1} \sum_{j=1}^{\mathrm{N}}\left|\lambda_{j}\right|^{n+1 / 2}=\delta_{n+1},
$$

а для $d=2$ (сумма под знаком интеграла отсутствует при $n=0)$

$$
\begin{aligned}
\pi^{-1}(-1)^{n} \int_{0}^{\infty}\left(\ln \left|D_{2}(\lambda+i 0)\right|-\sum_{j=1}^{n}(-1)^{j} \delta_{j} \lambda^{d / 2-1-j}\right) \lambda^{n-1 / 2} d \lambda & \\
& -(n+1 / 2)^{-1} \sum_{j=1}^{\mathrm{N}}\left|\lambda_{j}\right|^{n+1 / 2}=0 .
\end{aligned}
$$

Доказательство в существенном совпадает с доказательством теоремы 3.7 . В силу теоремы Коши

$$
\int_{\Gamma_{R, \varepsilon}} \ln D_{2}(z)(-z)^{n-1 / 2} d z=0
$$


где $\Gamma_{R, \varepsilon}$ - тот же контур, что и в (3.9). Интегралы по $C_{\varepsilon}^{ \pm}\left(\lambda_{j}\right)$ и $C_{\varepsilon}^{ \pm}(0)$ стремятся к нулю при $\varepsilon \rightarrow 0$. Таким образом, из (7.6) следует, что

$$
\begin{aligned}
2(-1)^{n} \int_{0}^{R} \ln \left|D_{2}(\lambda+i 0)\right| \lambda^{n-1 / 2} d \lambda-2 \pi(n+1 / 2)^{-1} \sum_{j=1}^{\mathrm{N}}\left|\lambda_{j}\right|^{n+1 / 2} & \\
& =i \int_{C_{R}} \ln D(z)(-z)^{n-1 / 2} d z .
\end{aligned}
$$

Асимптотические разложения при $R \rightarrow \infty$ обоих интегралов могут быть легко выведены из (7.3). При этом стремящиеся к бесконечности члены взаимно сокращаются. Остается перейти в (7.7) к пределу при $R \rightarrow \infty$.

Формулы (7.4) были получены, хотя и при существенно более ограничительных предположениях относительно $v(x)$, в работах [9], [12] и [10] соответственно для случаев $d=1$ и $d=3$. Насколько известно автору, формулы $(7.5)$ являются новыми.

Тождества следов целого порядка могут быть также получены методом доказательства теоремы 7.3 (при этом в (7.6) следует заменить $n-1 / 2$ на $n-1$ ), что требует, однако, более ограничительных предположений по сравнению с теоремой 7.1 .

\section{ЛитерАТУРА}

[1] S. Agmon and Y. Kannai, On the asymptotic behavior of spectral functions and resolvent kernels of elliptic operators, Israel J. Math., 5 (1967), 1-30.

[2] В. М. Бабич, Ю. О. Рапопорт, Асимптотика при мальх временах фундаментального решения задачи Коши для параболического уравнения второго порядка, Проблемы матем. физ., 7 (1974), 21-38.

[3] М. Ш. Бирман, М. Г. Крейн, K теории волновых операторов и операторов рассеяния, Докл. АН СССР, сер. матем., 144 (1962), 475-478.

[4] М. Ш. Бирман, М. З. Соломяк, Замечания о функции спектрального сдвига, Зап. научн. сем. ЛОМИ, 27 (1972), 33-46.

[5] М. Ш. Бирман, Д. Р. Яфаев, Функиия спектралъного сдвига. Работь М. Г. Крейна и их дальнейшее развитие, Алгебра и анализ, 4:5 (1992), 1-44.

[6] М. Ш. Бирман, Д. Р. Яфаев, Спектральные свойства матрищь рассеяния, Алгебра и анализ, 4:6 (1992), 1-27.

[7] J.-M. Bouclet, Trace formulae for relatively Hilbert-Schmidt perturbations, Asympt. Anal., 32:3-4 (2002), 257-291.

[8] В. С. Буслаев, Формуль следов и некоторые асимптотические оченки ядра резольвенты для оператора Шрёдингера в трехмерном пространстве, Проблемы матем. физ., 1 (1966), 82-101.

[9] В. С. Буслаев, Л. Д. Фаддеев, О формулах следов для сингулярного дифберенииального оператора Штурма-Лиувилля, Докл. АН СССР, сер. матем., 132 (1960), 13-16.

[10] Y. Colin de Verdière, Une formule de traces pour l'opérateur de Schrödinger dans $\mathbb{R}^{3}$, Ann. Sci. École Norm. Sup. (4), 14:1 (1981), 27-39.

[11] И. Ц. Гохберг, М. Г. Крейн, Введение в теорию линейных несамосопряженных операторов в гилъбертовом пространстве, Наука, М., 1965.

[12] В. Е. Захаров, Л. Д. Фаддеев, Уравнение Кортевега-де Фриса - вполне интегрируемая гамилътонова система, Функц. анализ и его прил., 5:4 (1971), 18-27. 
[13] M. Hitrik, I. Polterovich, Resolvent expansions and trace regularizations for Schrödinger operators, Contemp. Math., 327 (2003), 161-173.

[14] M. Hitrik, I. Polterovich, Regularized traces and Taylor expansions for the heat semigroup, J. London Math. Soc., 68:2 (2003), 402-418.

[15] A. Jensen, T. Kato, Spectral properties of Schrödinger operators and time-decay of the wave functions, Duke Math. J., 46:3 (1979), 583-611.

[16] S. Kantorovitz, $\mathbb{C}^{n}$-operational calculus, noncommutative Taylor formula and perturbation of semigroups, J. Funct. Anal., 113:1 (1993), 139-152.

[17] Л. С. Коплиенко, $K$ теории функиии спектрального сдвига, Проблемы матем. физ., 5 (1971), 62-72.

[18] Л. С. Коплиенко, Формула следа для возмущений неядерного типа, Сиб. матем. ж., 25:5 (1984), 62-71.

[19] М. Г. Крейн, О формуле следа в теории возмущений, Матем. сб., 33 (1953), 597626.

[20] М. Г. Крейн, Об определителях возмущения и формуле следов для унитарных и самосопряженных операторов, Докл. АН СССР, сер. матем., 144 (1962), 268-271.

[21] М. Г. Крейн, О некоторых новых исследованиях по теории возмущений, в кн.: Первая летняя матем. школа, Наукова думка, Киев, 1964, 103-187.

[22] S. T. Kuroda, Scattering theory for differential operators, J. Math. Soc. Japan, 25:1, 2 (1973), 75-104, 222-234.

[23] И. М. Лифшиц, Об одной задаче теории возмущений, УМН, 7:1 (1952), 171-180.

[24] R. Newton, Noncentral potentials: The generalized Levinson theorem and the structure of the spectrum, J. Math. Phys., 18:7 (1977), 1348-1357.

[25] В. В. Пеллер, Операторь Ганкеля в теории возмущений унитарных и самосопряженных операторов, Функц. анализ и его прил., 19:2 (1985), 37-51.

[26] I. Polterovich, Heat invariants of Riemannian manifolds, Israel J. Math., 119 (2000), 239-252.

[27] М. Рид, Б. Саймон, Методъ современной математической физики, т. 3, 4, Мир, Москва, 1982.

[28] D. Robert, Asymptotique à grande énergie de la phase de diffusion pour un potentiel, Asympt. Anal., 3:4 (1991), 301-320.

[29] D. Robert, Semiclassical asymptotics for the spectral shift function, in: Differential operators and spectral theory, Amer. Math. Soc. Transl., Ser. 2, vol. 189, Amer. Math. Soc., Providence, RI, 1999, 187-203.

[30] М. А. Шубин, Псевдодифференциальные операторы и спектральная теория, Наука, М., 1978.

[31] М. М. Скриганов, Равномерные координатные и спектралъные асимптотики решений задачи рассеяния для уравнения Шрёдингера, Зап. научн. сем. ЛОМИ, 69 (1977), 171-199.

[32] Д. Р. Яфаев, Замечание о теории рассеяния для возмущенного полигармонического оператора, Матем. заметки, 15 (1974), 445-454.

[33] Д. Р. Яфаев, Математическая теория рассеяния, Изд-во С.-Петербургского Университета, 1994.

[34] D. R. Yafaev, High energy asymptotics of the scattering amplitude for the Schrödinger equation, Proc. Indian Acad. Sci., Math. Sci., 112:1 (2002), 245-255.

[35] D. R. Yafaev, High energy and smoothness asymptotic expansion of the scattering amplitude, J. Funct. Anal., 202:2 (2003), 526-570.

IRMAR, University of Rennes-I,

Campus Beaulieu, FRANCE

Поступило в редакцию

e-mail: dimitri.yafaev@univ-rennes1.fr

21 декабря 2006 г. 